\title{
ON CERTAIN SOLUTIONS FOR CONFLUENT AND DOUBLE-CONFLUENT HEUN EQUATIONS
}

\author{
Léa Jaccoud El-Jaick and Bartolomeu D. B. Figueiredo \\ Centro Brasileiro de Pesquisas Físicas (CBPF) \\ Rua Dr. Xavier Sigaud, 150, CEP 22290-180, Rio de Janeiro, RJ, Brasil
}

\begin{abstract}
This paper examines some solutions for confluent and double-confluent Heun equations. In the first place, we review two Leaver's solutions in series of regular and irregular confluent hypergeometric functions for the confluent equation and introduce an additional expansion in series of irregular confluent hypergeometric functions. Then, we find the conditions under which one of these solutions can be written as a linear combination of the others. In the second place, by means of limiting procedures we generate solutions for the double-confluent equation as well as for special limits of both the confluent and double-confluent equations. Finally, we present problems which are ruled by each of these four equations and establish relations among Heun equations and quasi-exactly solvable problems.
\end{abstract}

\section{Introduction}

This paper is concerned with solutions of Heun equations and possible applications of such solutions. We consider only the confluent and the double-confluent Heun equations (CHE and DCHE, respectively), and one limiting case of each of these. The solutions for the CHE come directly from the differential equation, while the solutions for the other equations are obtained from the solutions of the CHE by limiting processes. Initially, we briefly discuss each of these equations and their connections; some more details are found in previous works [14, 15, 16, 25. Then, we outline the main features of the solutions and the structure of the paper.

The CHE [10, 11, 34], also known as generalised spheroidal wave equation [42, 43], in the form used by Leaver 25 ] reads $(\omega \neq 0)$

$$
z\left(z-z_{0}\right) \frac{d^{2} U}{d z^{2}}+\left(B_{1}+B_{2} z\right) \frac{d U}{d z}+\left[B_{3}-2 \eta \omega\left(z-z_{0}\right)+\omega^{2} z\left(z-z_{0}\right)\right] U=0,
$$

where $B_{i}, \eta$ and $\omega$ are constants and $z=0$ and $z=z_{0}$ are regular singular points with indicial exponents $\left(0,1+B_{1} / z_{0}\right)$ and $\left(0,1-B_{2}-B_{1} / z_{0}\right)$, respectively. At the irregular point $z=\infty$ the behaviour of the solutions, obtained from the normal Thomé solutions [25, 33], is given by

$$
\lim _{z \rightarrow \infty} U(z) \sim e^{ \pm i \omega z} z^{\mp i \eta-\left(B_{2} / 2\right)} .
$$

The singularity parameter $z_{0}$ may take any value and, when $z_{0}=0$, the CHE gives the following DCHE with five parameters [25]

$$
z^{2} \frac{d^{2} U}{d z^{2}}+\left(B_{1}+B_{2} z\right) \frac{d U}{d z}+\left(B_{3}-2 \eta \omega z+\omega^{2} z^{2}\right) U=0,\left(B_{1} \neq 0, \omega \neq 0\right),
$$

where now $z=0$ and $z=\infty$ are both irregular singularities $\left(B_{1}=0\right.$ and/or $\omega=0$ are degenerate cases [15]). At $z=\infty$ the behaviour is again given by Eq. (2), while at $z=0$ the normal Thomé solutions afford

$$
\lim _{z \rightarrow 0} U(z) \sim 1, \text { or } \lim _{z \rightarrow 0} U(z) \sim e^{B_{1} / z} z^{2-B_{2}} .
$$


The CHE and the DCHE admit a limit which changes the nature of the irregular singularity at $z=\infty$, keeping unaltered the other singular points. This limit is obtained by letting that [15, 16]

$$
\omega \rightarrow 0, \quad \eta \rightarrow \infty, \text { such that } 2 \eta \omega=-q, \quad \text { (Whittaker-Ince limit) }
$$

where $q$ is a constant. It is called Whittaker-Ince limit because Whittaker and Ince have used a similar procedure to get the Mathieu equation (12) from the WhittakerHill equation (11) [19, 21]. The Whittaker-Ince limit of the CHE is

$$
z\left(z-z_{0}\right) \frac{d^{2} U}{d z^{2}}+\left(B_{1}+B_{2} z\right) \frac{d U}{d z}+\left[B_{3}+q\left(z-z_{0}\right)\right] U=0, \quad(q \neq 0)
$$

(if $q=0$ this equation can be transformed into a hypergeometric equation), while the Whittaker-Ince limit of the DCHE is

$$
z^{2} \frac{d^{2} U}{d z^{2}}+\left(B_{1}+B_{2} z\right) \frac{d U}{d z}+\left(B_{3}+q z\right) U=0, \quad\left(q \neq 0, B_{1} \neq 0\right)
$$

(if $q=0$ and/or $B_{1}=0$ the equation degenerates into a confluent hypergeometric equation or simpler equations [15]). Eqs. ([6) and (7) differ from the CHE and DCHE, respectively, by the behaviour of their solutions at the irregular point $z=\infty$, which now is obtained from the subnormal Thomé solutions [33], namely,

$$
\lim _{z \rightarrow \infty} U(z) \sim e^{ \pm 2 i \sqrt{q z}} z^{(1 / 4)-\left(B_{2} / 2\right)}
$$

in contrast with the behaviour of original equations (normal Thomé solutions). Eq. (77) also results when we take $z_{0}=0$ in Eq. (6).

The preceding equations and their connections are summarised in the following diagram which is a modified version of a diagram given in [16. The upper boxes display the CHE and the DCHE. The lower boxes show the Whittaker-Ince limits corresponding to the CHE and DCHE, respectively.

$$
\begin{aligned}
& \begin{array}{|l|}
z\left(z-z_{0}\right) \frac{d^{2} U}{d z^{2}}+\left(B_{1}+B_{2} z\right) \frac{d U}{d z}+ \\
{\left[B_{3}-2 \eta \omega\left(z-z_{0}\right)+\omega^{2} z\left(z-z_{0}\right)\right] U=0}
\end{array} \stackrel{z_{0} \rightarrow 0}{\Rightarrow} \begin{array}{l}
z^{2} \frac{d^{2} U}{d z^{2}}+\left(B_{1}+B_{2} z\right) \frac{d U}{d z}+ \\
\left(B_{3}-2 \eta \omega z+\omega^{2} z^{2}\right) U=0
\end{array} \\
& \Downarrow \quad(\omega \rightarrow 0 \text { and } \eta \rightarrow \infty \text {, such that } 2 \eta \omega=-q) \quad \Downarrow
\end{aligned}
$$

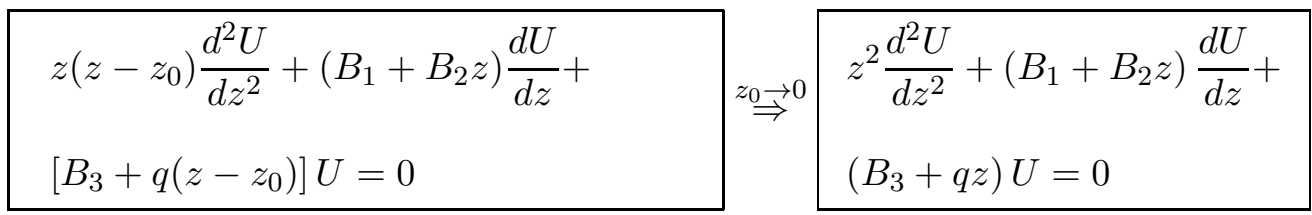

These connections among equations having different types of singularities become fully effective only when solutions of the CHE admit both the Leaver and the Whittaker-Ince limits. Counter-examples are provided by Hylleraas [20] and Jaffé's [22] solutions which admit none of these limits, as we can see by using Leaver's form for such solutions [25].

Now we introduce the Whittaker-Hill and the Mathieu equations which are particular cases of both the CHE and the DCHE [10]. A trigonometric (hyperbolic) form of the Whittaker-Hill equation (WHE) is [2, 21]

$$
\frac{d^{2} W}{d u^{2}}+\kappa^{2}\left[\vartheta-\frac{1}{8} \xi^{2}-(p+1) \xi \cos (2 \kappa u)+\frac{1}{8} \xi^{2} \cos (4 \kappa u)\right] W=0, \quad(\mathrm{WHE})
$$


If $u$ is a real variable, this equation represents the usual WHE when $\kappa=1$ and the modified WHE when $\kappa=i$. On the other hand, the Mathieu equation has the form [30.

$$
\frac{d^{2} w}{d u^{2}}+\sigma^{2}\left[a-2 k^{2} \cos (2 \sigma u)\right] w=0, \quad \text { (Mathieu equation) }
$$

where $\sigma=1$ or $\sigma=i$ for the Mathieu or modified Mathieu equation, respectively. Some details about solutions for the WHE and Mathieu equation regarded as CHE or DCHE are given in Ref. [16]. The Mathieu equation is also a particular case of equation (7) as shown in section 3. Incidentally, the original Whittaker-Ince limit [19, 21] is obtained when $\xi \rightarrow 0, p \rightarrow \infty$ so that $p \xi=2 k^{2}, \kappa=\sigma$ and $\vartheta=a$ in the WHE. This gives the Mathieu equation.

On the other hand, the solutions for the Heun equations, and in particular for CHE and DCHE, take one of the following forms 34]

$$
\sum_{n} a_{n} f_{n}(z)=\sum_{n=-\infty}^{\infty} a_{n} f_{n}(z), \quad \sum_{n=0}^{\infty} a_{n} f_{n}(z), \quad \sum_{n=0}^{N} a_{n} f_{n}(z),
$$

where the series coefficients $a_{n}$ satisfy three-term or higher order recurrence relations, $f_{n}(z)$ is a function of the independent variable $z, N$ is a non-negative integer and the symbol $\sum_{n}$ holds for summation running from negative to positive infinity. These are called, respectively, two-sided infinite series, one-sided infinite series and finite series. The finite series are also known as quasi-polynomial solutions, quasi-algebraic solutions or Heun polynomials.

Expansions in two-sided infinite series are necessary to assure the series convergence when there is no free constant in the Heun equations. Thus, all the parameters of the CHE and DCHE which rule the time-dependence of Klein-Gordon and Dirac test-fields in some Friedmannian spacetimes [6, 14] are determined from conditions imposed on the spatial part of the wave functions [35, 36]. Similarly, in the scattering problem of ions by a finite dipole [25] or by polarisable targets [7, 15] all the parameters of the radial Schrödinger equation are known.

When some parameters of the Heun equations assume special values, one-sided infinite series truncate on the right giving expansions in finite series. These Heun polynomials may be useful to get solutions for quasi-exactly solvable (QES) problems [37, 38, 39, 40]. In effect, a problem is QES if admits solutions given by finite-series whose coefficients necessarily satisfy three-term or higher order recurrence relations [23]; in contrast, a problem is exactly solvable if its solutions are given by (generalised) hypergeometric functions. Actually, there are QES potentials for which the Schrödinger equation leads to the CHE and DCHE as well as to the general, biconfluent and triconfluent Heun equations (see Appendix A).

Excepting possibly the Heun polynomials, in general the solutions for the Heun equations do not converge for the entire range of the independent variable. Then, it is necessary to consider two or more solutions converging over different domains and having the appropriate behaviours at the singular points. It is also necessary to take into account the transformation rules which generate new solutions from a known solution (these rules result from substitutions of variables which preserve the form of the Heun equations but modify their parameters).

We will start with a set of three solutions for the CHE, represented by two-sided infinite series which have coefficients that satisfy three-term recurrence relations. These solutions admit both the Leaver and the Whittaker-Ince limits and so we can generate sets of solutions for all the equations discussed above. Solutions obtained from this set by means of transformation rules are given in sections 2.4 and 3.3. 
More precisely, in Sec. 2 we take two Leaver's solutions in series of regular and irregular confluent hypergeometric functions for $\mathrm{CHE}$ and introduce another expansion in series of irregular confluent hypergeometric functions - see the solutions given in Eqs. (33a) and the recurrence relations (33b) and (33c). The expansion in series of regular functions converges for any $z$, whereas the two expansions in series of irregular functions converge for $|z|>\left|z_{0}\right|$. From the properties of the three-term recurrence relations and of the hypergeometric functions, we shall find conditions which permit to write one solution as a linear combination of the others in the region $|z|>\left|z_{0}\right|$. These conditions also assure that the series coefficients of the three solutions are proportional to each other.

We advance that the convergence of the expansion in regular functions over the entire complex plane does not dispense with the expansions in series of irregular functions. In fact, if the expansion in regular functions is a linear combination of the others, its behaviour at $z=\infty$ must be given by a linear combination of the two behaviours given in equation (2), since each expansion in irregular functions corresponds to one of those behaviours. However, if for instance $i \omega z$ is real, one of the exponentials $\exp ( \pm i \omega z)$ goes to infinity. In this case, to get a solution finite when $z \rightarrow \infty$ we have to use one of the expansions in irregular functions.

The solutions for the limits of the $\mathrm{CHE}$, given in the above diagram, are obtained by the same procedure used in Ref. [16, where a set having only two solutions in terms of one-sided series of confluent hypergeometric functions was considered. Thus, in Sec. 3 we find a set of three expansions in series of Bessel functions for Whittaker-Ince limit (6) of the CHE, the three solutions possessing exactly the same series coefficients. In Sec. 4 we find that the solutions for the DCHE are again given by series of confluent hypergeometric, while the solutions for Whittaker-Ince limit of the DCHE are given by series of Bessel functions once more.

In summary, in Sec. 2 we deal with the CHE, in Sec. 3 with the Whittaker-Ince limit (6) of the CHE, and in Sec. 4 with the DCHE and its limit (7). Sec. 5 presents some conclusions and points to problems which are ruled by the equations of the above diagram. Appendix A shows some relations among Heun equations and quasiexactly solvable problems, while Appendix B gives some properties of the confluent hypergeometric functions.

\section{The Confluent Heun Equation (CHE)}

In this section we review the two Leaver solutions in series of regular and irregular confluent hypergeometric functions for the confluent Heun equation [25] and introduce the extra expansion in series of irregular confluent hypergeometric functions. The fact that the expansion in terms of regular functions converges for any $z$ (the expansions in terms of irregular functions converge for $\left.|z|>\left|z_{0}\right|\right)$ distinguishes the present solutions from the Leaver expansions in series of Coulomb wave functions [25] since the latter converge only for $|z|>\left|z_{0}\right|$ (if the series is doubly infinite).

Firstly, in Sec. 2.1, we recall some features of three-term recurrence relations for the series coefficients and supply properties of the confluent hypergeometric functions which enter the solutions for the CHE and DCHE. We also write down a BarberHassé solution in power series since this is important to obtain finite-series solutions as well as to cover the cases in which the expansions in series of regular confluent hypergeometric functions are not valid.

In Sec. 2.2 we analyse the set constituted by the expansions in series of confluent hypergeometric functions. This is called the fundamental set of solutions because, by means of the transformation rules it originates other sets of solutions for the CHE and, by way of limiting processes, it affords sets of solutions for the other equations 
given in the schema of the first section. We find the conditions under which one solution of the fundamental set is given as a linear combination of the others. After that, we truncate the two-sided series from below in order to get one-sided series solutions as well. In Sec. 2.3, we discuss the convergence of new expansion in series of irregular confluent hypergeometric functions and, finally, in Sec. 2.4 we show how we can generate new sets of solutions by using the transformation rules for the CHE.

\subsection{General remarks and the Barber-Hassé expansions}

If the coefficients of one-sided series solutions are denoted by $b_{n}$, then the threeterm recurrence relations have the form

$$
\alpha_{0} b_{1}+\beta_{0} b_{0}=0, \quad \alpha_{n} b_{n+1}+\beta_{n} b_{n}+\gamma_{n} b_{n-1}=0 \quad(n \geq 1)
$$

where $\alpha_{n}, \beta_{n}$ and $\gamma_{n}$ depend on the parameters of the differential equation. This system of homogeneous linear equations has nontrivial solutions for $b_{n}$ only if the determinant of the respective infinite tridiagonal matrix vanishes. This demands the presence of some arbitrary parameter in the differential equation. Equivalently, these recurrence relations imply a characteristic equation given by the infinite continued fraction [25]

$$
\beta_{0}=\frac{\alpha_{0} \gamma_{1}}{\beta_{1}-} \frac{\alpha_{1} \gamma_{2}}{\beta_{2}-} \frac{\alpha_{2} \gamma_{3}}{\beta_{3}-} \cdots
$$

which must be satisfied in order to assure the series convergence.

If $\gamma_{n}=0$ for some $n=N+1$, where $N$ is a non-negative integer, the onesided series terminates at $n=N$ and, consequently, gives a finite-series solution with $0 \leq n \leq N$ [2]. Thus the recurrence relations can be written in the form

$$
\left(\begin{array}{ccccccc}
\beta_{0} & \alpha_{0} & 0 & \cdots & & & 0 \\
\gamma_{1} & \beta_{1} & \alpha_{1} & & & & \\
0 & \gamma_{2} & \beta_{2} & \alpha_{2} & & & \\
\vdots & & & & & & \\
& & & & & & \\
& & & & \gamma_{N-1} & \beta_{N-1} & \alpha_{N-1} \\
0 & \cdots & & & 0 & \gamma_{N} & \beta_{N}
\end{array}\right)\left(\begin{array}{l}
b_{0} \\
b_{1} \\
b_{2} \\
\vdots \\
\\
\\
b_{N-1} \\
b_{N}
\end{array}\right)=0 .
$$

If the elements $\alpha_{i}, \beta_{i}$ and $\gamma_{i}$ of the previous matrix are real and if

$$
\alpha_{i} \gamma_{i+1}>0, \quad 0 \leq i \leq N-1,
$$

then all the $N+1$ roots of its determinant are real and different [2]. This theorem is important to determine a part of the energy spectra in the case of quasi-exact potentials. On the other hand, if $\alpha_{n}=0$ for some $n=N$, the series begins at $n=N+1$, but in this case one may set $n=m+N+1$ and rename the series coefficients in order to obtain a series beginning at $m=0$.

Now, suppose that there is a second solution with coefficients $c_{n}$ satisfying

$$
\tilde{\alpha}_{0} c_{1}+\beta_{0} c_{0}=0, \quad \tilde{\alpha}_{n} c_{n+1}+\beta_{n} c_{n}+\tilde{\gamma}_{n} c_{n-1}=0 \quad(n \geq 1),
$$

where $\beta_{n}$ is the same as in Eq. (14). Then, if

$$
\tilde{\alpha}_{n} \tilde{\gamma}_{n+1}=\alpha_{n} \gamma_{n+1},
$$

it follows from Eq. (15) that both solutions have the same characteristic equation if $n$ takes the same values in both series: in these circumstances, $b_{n}$ and $c_{n}$ in general 
are proportional to each other. Such proportionality requires the same range for $n$ in Eqs. (14) and (18) because there are cases in which the relation (19) is formally satisfied, but one solution is given by a finite series while the other is given by an infinite series. In these cases one series breaks off on the right and the other on the left.

The previous remarks can be extended to doubly infinite (or two-sided) series. These expansions present a parameter $\nu$ which must be determined from a characteristic equation if there is no free parameter in the differential equation, or can be chosen at will if there is a free constant. The recurrence relations for the series coefficients $b_{n}$ now take the form

$$
\alpha_{n} b_{n+1}+\beta_{n} b_{n}+\gamma_{n} b_{n-1}=0, \quad(-\infty<n<\infty)
$$

where $\alpha_{n}, \beta_{n}, \gamma_{n}$ and $b_{n}$ depend on the parameters of the differential equation as well as on $\nu$. These recurrence relations lead to the characteristic equation [25]

$$
\beta_{0}=\frac{\alpha_{-1} \gamma_{0}}{\beta_{-1}-} \frac{\alpha_{-2} \gamma_{-1}}{\beta_{-2}-} \frac{\alpha_{-3} \gamma_{-2}}{\beta_{-3}-} \cdots+\frac{\alpha_{0} \gamma_{1}}{\beta_{1}-} \frac{\alpha_{1} \gamma_{2}}{\beta_{2}-} \frac{\alpha_{2} \gamma_{3}}{\beta_{3}-} \cdots .
$$

If there is a second doubly infinite series having the recurrence relation $\tilde{\alpha}_{n} c_{n+1}+$ $\beta_{n} c_{n}+\tilde{\gamma}_{n} c_{n-1}=0$ such that the condition (19) is fulfilled, then both solutions satisfy the same characteristic equation (20b). The two series are really doubly infinite if neither the coefficients of $b_{n+1}$ and $c_{n+1}$ nor the coefficients of $b_{n-1}$ and $c_{n-1}$ vanish, since such conditions assure that the summation extends from negative to positive infinity in both solutions. This requires that

$$
\alpha_{n}, \tilde{\alpha}_{n}, \gamma_{n} \text { and } \tilde{\gamma}_{n} \text { do not vanish for any } n,
$$

a requirement which imposes constraints on the parameters of differential equation and on the characteristic parameter $\nu$, in the case of two-sided series. These conditions will be useful for studying the sets of two-sided solutions of the CHE and DCHE. In addition, if we choose $\nu$ such that $\alpha_{-1}=\tilde{\alpha}_{-1}=0$, then the series are truncated on the left since the summation begins at $n=0$. Thus, we have

$$
\alpha_{-1}=\tilde{\alpha}_{-1}=0 \quad \Rightarrow \quad \text { one-sided series with } n \geq 0 .
$$

Other restrictions on $\nu$ and on the parameters of the Heun equations come from the properties of the special functions used to construct the series solutions. Thus, let us consider expansions in series of regular and irregular confluent hypergeometric functions for the CHE, denoted by $\Phi(a, c ; y)$ and $\Psi(a, c ; y)$ respectively. These are solutions of the confluent hypergeometric equation 12

$$
y \frac{d^{2} \varphi}{d y^{2}}+(c-y) \frac{d \varphi}{d y}-a \varphi=0,
$$

where the parameters $a$ and $c$ will depend on summation index $n$, on the parameters of the Heun equations and also on the characteristic parameter $\nu$ in the case of two-sided infinite series. In fact, the following four solutions for Eq. (23)

$$
\begin{array}{ll}
\varphi_{n}^{1}(y)=\Phi(a, c ; y), & \varphi_{n}^{2}(y)=\Psi(a, c ; y), \\
\varphi_{n}^{3}(y)=e^{y} y^{1-c} \Phi(1-a, 2-c ;-y), & \varphi_{n}^{4}(y)=e^{y} y^{1-c} \Psi(1-a, 2-c ;-y)
\end{array}
$$

are all of them defined and distinct only if $c$ is not an integer [12]. Furthermore, if

$$
a, c \text { and } c-a \text { are not integer , }
$$


then any two of the solutions (24) form a fundamental system of solutions for confluent hypergeometric equation [12]. The formula

$$
\Psi(a, c ; y)=\frac{\Gamma(1-c)}{\Gamma(a-c+1)} \Phi(a, c ; y)+\frac{\Gamma(c-1)}{\Gamma(a)} y^{1-c} \Phi(a-c+1,2-c ; y),
$$

gives the analytic continuation of $\Psi$ in terms of $\Phi$. The expression of $\Phi$ in terms of $\Psi$ is obtained from the previous one by using the relation $\Gamma(z) \Gamma(1-z)=\pi / \sin (\pi z)$. One finds [12],

$$
\Phi(a, c ; y)=\frac{e^{i \pi a \varepsilon} \Gamma(c)}{\Gamma(c-a)} \Psi(a, c ; y)+\frac{e^{i \pi \varepsilon(a-c)} \Gamma(c)}{\Gamma(a)} e^{y} \Psi(c-a, c ;-y), \quad(\varepsilon= \pm 1)
$$

where the plus or minus signs are to be taken throughout following the conventions

$$
\varepsilon=1 \text { if }(-1)=e^{i \pi}, \quad \varepsilon=-1 \text { if }(-1)=e^{-i \pi} \Rightarrow(-1)^{c}=e^{i \pi c}, \quad(-1)^{c}=e^{-i \pi c}
$$

(by setting $c=1 / 2$ we see that $\varepsilon=1$ if we take $\sqrt{-1}=i$, and $\varepsilon=-1$ if $\sqrt{-1}=-i$ ). The relation (26) allows writing $\Phi(a, c ; y)$ as a combination of a regular and an irregular confluent hypergeometric functions; analogously, Eq. (27) gives $\Psi(a, c ; y)$ in terms of regular and irregular functions.

The general form of the expansions in series of confluent hypergeometric functions for the CHE and DCHE is

$$
U\left(\varphi_{n}^{i}(y)\right)=e^{-i \omega z} \sum_{n} \pi_{n}^{i} \varphi_{n}^{i}(y),
$$

where $\pi_{n}^{i}$ denotes the series coefficients. In the fundamental set of solutions (33a) the argument of the hypergeometric functions $\varphi_{n}^{i}$ is $y=2 i \omega z$, and the parameters are $a=\left(B_{2} / 2\right)-i \eta$ and $c=n+\nu+B_{2}$. We use only the functions $\varphi_{n}^{1}(y), \varphi_{n}^{2}(y)$ and $\varphi_{n}^{4}(y)$ because $\varphi_{n}^{3}(y)$ would lead to a solution whose domain of convergence excludes all the singular points of the equation. As $\varphi_{n}^{3}(y)$ is discarded, the formula (27) is the only one necessary to connect the three hypergeometric functions. Consequently, the conditions (25) are replaced by

$$
a, c \text { and } c-a \text { are not zero or negative integers. }
$$

The present conditions combined with conditions (21) allow to use Eq. (27) in order to write one solution of the CHE or DCHE as a linear combination of the others.

Now we write down a Barber-Hassé solutions in series of $\left(z-z_{0}\right)$ for the CHE [3, 16, 25]. They converge for finite values of $z$. The first solution is

$$
U_{1}^{\text {barber }}(z)=e^{i \omega z} \sum_{n=0}^{\infty} a_{n}^{(1)}\left(z-z_{0}\right)^{n}, \quad(|z|=\text { finite })
$$

where recurrence relations for the coefficients are given by $\left(a_{-1}^{(1)}=0\right)$

$$
\begin{aligned}
& z_{0}\left(n+B_{2}+\frac{B_{1}}{z_{0}}\right)(n+1) a_{n+1}^{(1)}+\left[n\left(n+B_{2}-1+2 i \omega z_{0}\right)\right. \\
& \left.+B_{3}+i \omega z_{0}\left(B_{2}+\frac{B_{1}}{z_{0}}\right)\right] a_{n}^{(1)}+2 i \omega\left(n+i \eta+\frac{B_{2}}{2}-1\right) a_{n-1}^{(1)}=0 .
\end{aligned}
$$

This expansion will provide solutions to the CHE for the case in which the Leaver expansion in regular hypergeometric functions, given in the following, is not valid, and admits both the Leaver and the Whittaker-Ince limits. Furthermore, it gives finiteseries solutions with $0 \leq n \leq N$ when $i \eta+B_{2} / 2=-N$. However, the Whittaker-Ince 
limit of this Barber-Hassé solution does not admit finite-series solutions since the coefficient of $a_{n-1}$ is a constant: $2 i \omega\left(n+i \eta+B_{2} / 2-1\right) \rightarrow q$.

We may generate a group containing 16 sets of solutions for the CHE by applying to the previous solution the transformation rules given in Sec. 2.4. These solutions for the CHE give solutions for the Whittaker-Hill equation (WHE). In effect, if $U(z)$ symbolises the solutions for the CHE, the solutions $W(u)$ for the WHE (11) are obtained by writing [16]

$$
W(u)=U(z), \quad z=\cos ^{2}(\kappa u), \quad(\kappa=1, i)
$$

where the parameters of $U(z)$ are given in terms of the parameters of the WHE by

$$
z_{0}=1, \quad B_{1}=-\frac{1}{2}, \quad B_{2}=1, \quad B_{3}=\frac{1}{4}[(p+1) \xi-\vartheta], \quad i \omega=\frac{\xi}{2}, \quad i \eta=\frac{p+1}{2} .
$$

For the WHE, the Barber-Hassé solutions become even or odd solutions with respect to the change of the sign of $u[16]$.

\subsection{The fundamental set of solutions}

First we examine the two-sided infinite series solutions and then we obtain the one-sided series. It is worth advancing that the solutions in terms of regular confluent hypergeometric functions $\Phi(a, c ; y)$ are not valid when the first parameter $a$ is zero or a negative integer. This statement is true only if the solutions are written so that the parameter $a$ does not depend on the summation index $n$, since the Kummer relation (B.1) gives another representation in which the first parameter of $\Phi(a, c ; y)$ depends on $n$.

Defining the function $\tilde{\Phi}(a, b ; y)$ by [25]

$$
\tilde{\Phi}(a, c ; y)=\frac{\Gamma(c-a)}{\Gamma(c)} \Phi(a, c ; y)=\frac{\Gamma(c-a)}{\Gamma(c)}\left[1+\frac{a}{1 ! c} y+\frac{a(a+1)}{2 ! c(c+1)} y^{2}+\cdots\right],
$$

the fundamental set (28) reads

$$
\begin{aligned}
U_{1}(z)= & e^{-i \omega z} \sum_{n}(-1)^{n} b_{n}^{(1)} \tilde{\Phi}\left(\frac{B_{2}}{2}-i \eta, n+\nu+B_{2} ; 2 i \omega z\right), \\
U_{1}^{\infty}(z)= & e^{-i \omega z} \sum_{n}(-1)^{n} b_{n}^{(1)} \Psi\left(\frac{B_{2}}{2}-i \eta, n+\nu+B_{2} ; 2 i \omega z\right), \\
\bar{U}_{1}^{\infty}(z)= & e^{i \omega z} \times \\
& \sum_{n} c_{n}^{(1)}(-2 i \omega z)^{1-n-\nu-B_{2}} \Psi\left(1+i \eta-\frac{B_{2}}{2}, 2-n-\nu-B_{2} ;-2 i \omega z\right)
\end{aligned}
$$

where the recurrence relations for $b_{n}^{(1)}$ and $c_{n}^{(1)}$ are

$$
\begin{aligned}
& (n+\nu+1)\left(n+\nu+i \eta+\frac{B_{2}}{2}\right) b_{n+1}^{(1)}+\left[(n+\nu)\left(n+\nu+B_{2}-1+2 i \omega z_{0}\right)\right. \\
& \left.+B_{3}+i \omega z_{0}\left(B_{2}+\frac{B_{1}}{z_{0}}\right)\right] b_{n}^{(1)}+2 i \omega z_{0}\left(n+\nu+B_{2}+\frac{B_{1}}{z_{0}}-1\right) b_{n-1}^{(1)}=0 .
\end{aligned}
$$

and

$$
\begin{aligned}
& (n+\nu+1) c_{n+1}^{(1)}+\left[(n+\nu)\left(n+\nu+B_{2}-1+2 i \omega z_{0}\right)+i \omega z_{0}\left(B_{2}+\frac{B_{1}}{z_{0}}\right)\right. \\
& \left.+B_{3}\right] c_{n}^{(1)}+2 i \omega z_{0}\left(n+\nu+B_{2}+\frac{B_{1}}{z_{0}}-1\right)\left(n+\nu+i \eta+\frac{B_{2}}{2}-1\right) c_{n-1}^{(1)}=0
\end{aligned}
$$


Apart from a multiplicative constant, the coefficients $b_{n}^{(1)}$ and $c_{n}^{(1)}$ are connected by

$$
c_{n}^{(1)}=\Gamma\left(n+\nu+i \eta+\frac{B_{2}}{2}\right) b_{n}^{(1)},
$$

provided that the argument of the gamma function is not zero or negative integer.

The solutions $U_{1}(z)$ and $U_{1}^{\infty}(z)$ have been taken from Eqs. (166) and (167) of Leaver's paper [25] with $\nu$ replaced by $\nu+B_{2}$. On the other hand, by using a Kummer relation given in Eq. (B.1), $\bar{U}_{1}^{\infty}(z)$ is rewritten as

$$
\bar{U}_{1}^{\infty}(z)=e^{i \omega z} \sum_{n} c_{n}^{(1)} \Psi\left(n+\nu+i \eta+\frac{B_{2}}{2}, n+\nu+B_{2} ;-2 i \omega z\right) .
$$

Then, it becomes clear that this solution can be obtained by substituting $n+\nu$ for $n$ in the one-sided solution given in Eq. (33a) of Ref. [16] and by allowing that the summation runs from negative to positive infinity. The solution $U_{1}$ converges for any $z$ [25], while both $U_{1}^{\infty}$ and $\bar{U}_{1}^{\infty}$ converge for $|z|>\left|z_{0}\right|$. From the fact that $\Phi(a, c ; 0)=1$ and from Eq. (B.2), it follows that

$$
\lim _{z \rightarrow 0} U_{1}(z) \sim 1, \quad \lim _{z \rightarrow \infty} U_{1}^{\infty}(z) \sim e^{-i \omega z} z^{i \eta-\frac{B_{2}}{2}}, \quad \lim _{z \rightarrow \infty} \bar{U}_{1}^{\infty}(z) \sim e^{i \omega z} z^{-i \eta-\nu-\frac{B_{2}}{2}} .
$$

Thus, two different behaviours at $z=\infty$ are included in the solutions belonging to the same set. Notice that the parameter $\nu$ does not appear in Eq. (2) because this was obtained from solutions given by one-sided series (Thomé solutions).

Since we are dealing with three solutions for a second order linear differential equation, now we establish the conditions to get one of these as a linear combination of the others in a domain where the three solutions are valid. From the recurrence relations (33b) and (33c) we find that the three series are really doubly infinite if

$$
\nu, \nu+B_{2}+\frac{B_{1}}{z_{0}} \text { and } \nu+i \eta+\frac{B_{2}}{2} \text { are not integers, }
$$

since under these conditions neither the coefficients of $b_{n}^{(1)}$ and $c_{n}^{(1)}$ nor the coefficients of $b_{n-1}^{(1)}$ and $c_{n-1}^{(1)}$ vanish, that is to say, the series do not truncate on the left or on the right. The last condition also assures that the series coefficients are linked by Eq. (34) and in turn this implies that there is a unique characteristic equation. If, in addition to conditions (37a), the conditions (29) are also satisfied, that is, if

$$
n+\nu+B_{2}, \frac{B_{2}}{2}-i \eta \text { and } n+\nu+i \eta+\frac{B_{2}}{2} \text { are not zero or negative integers, }
$$

then Eq. (27) may be used to prove that any of the three solutions is a linear combination of the others in a region where the three solutions are valid $\left(|z|>\left|z_{0}\right|\right)$. Since $n$ runs from $-\infty$ to $\infty$, the above conditions are equivalent to

$$
\nu+B_{2}, \nu+i \eta+\frac{B_{2}}{2} \text { are not integers; } \frac{B_{2}}{2}-i \eta \text { is not zero or negative integer, }
$$

which repeat one of the conditions (37a).

In short, to express one solution in terms of the others, the three solutions must be given by two-sided series and the formula (27) for analytic continuation of the hypergeometric functions must hold. These are the general conditions which may be applied to any set of solutions generated from the first set through the transformation rules of the CHE. In fact, they are equivalent to the conditions (21) and (29).

If $U_{1}$ is a superposition of $U_{1}^{\infty}$ and $\bar{U}_{1}^{\infty}$ in the common domain of convergence $\left(|z|>\mid z_{0}\right)$, then the behaviour of $U_{1}$ when $z \rightarrow \infty$ must be given by a combination of the behaviours of $U_{1}^{\infty}$ and $\bar{U}_{1}^{\infty}$. However, for certain problems, one of the expansions 
in irregular functions may be inadequate when $z \rightarrow \infty$ and, consequently, $U_{1}$ becomes inappropriate as well.

Only the restriction on the values of $\left(B_{2} / 2\right)-i \eta$ cannot be satisfied by a convenient choice of $\nu$. This restriction also arises if we consider the solution $U_{1}(z)$ by itself, disregarding its connection with the other solutions. In fact, if $\left(B_{2} / 2\right)-i \eta=-m$ $(m=0,1,2, \cdots)$, the hypergeometric function $\Phi(a, c ; y)$ which appears in $U_{1}$ becomes a polynomial of degree $m$ with respect to its argument [1] and, then, the summation from negative to positive infinity is meaningless. The solution

$$
U_{1}^{\mathrm{p}}(z)=e^{-i \omega z} \sum_{n=0}^{\infty} d_{n}^{(1)}\left(z-z_{0}\right)^{n}, \quad(|z|=\text { finite })
$$

where recurrence relations for the coefficients are $\left(d_{-1}^{(1)}=0\right)$

$$
\begin{aligned}
& z_{0}\left(n+B_{2}+\frac{B_{1}}{z_{0}}\right)(n+1) d_{n+1}^{(1)}+\left[n\left(n+B_{2}-1-2 i \omega z_{0}\right)\right. \\
& \left.+B_{3}-i \omega z_{0}\left(B_{2}+\frac{B_{1}}{z_{0}}\right)\right] d_{n}^{(1)}-2 i \omega\left(n-i \eta+\frac{B_{2}}{2}-1\right) d_{n-1}^{(1)}=0,
\end{aligned}
$$

takes the place of $U_{1}$ when $\left(B_{2} / 2\right)-i \eta=-m$. Notice that $U_{1}^{\mathrm{p}}$ was obtained from the Barber-Hassé expansion (30a) by substituting $(-\omega,-\eta)$ for $(\omega, \eta)$. Furthermore, even if $\left(B_{2} / 2\right)-i \eta=-m$, by means of the transformation rules we can find two-sided series expansions in terms of regular confluent hypergeometric functions which hold for this case.

Now we consider the one-sided series solutions. From the recurrence relations $(33 \mathrm{~b})$ and (33c) we see that for truncating the three solutions on the left at $n=0$ the only choice of $\nu$ common to the three solutions is $\nu=0$ - see Eq. (22). We rewrite these one-sided series solutions as

$$
\begin{aligned}
& U_{1}(z)=e^{-i \omega z} \sum_{n=0}^{\infty} \frac{(-1)^{n} c_{n}^{(1)}}{\Gamma\left(n+B_{2}\right)} \Phi\left(\frac{B_{2}}{2}-i \eta, n+B_{2} ; 2 i \omega z\right), \\
& U_{1}^{\infty}(z)=e^{-i \omega z} \sum_{n=0}^{\infty} b_{n}^{(1)} \Psi\left(\frac{B_{2}}{2}-i \eta, n+B_{2} ; 2 i \omega z\right), \\
& \bar{U}_{1}^{\infty}(z)=e^{i \omega z} \sum_{n=0}^{\infty} c_{n}^{(1)} \Psi\left(n+i \eta+\frac{B_{2}}{2}, n+B_{2} ;-2 i \omega z\right),
\end{aligned}
$$

where the recurrence relations are $\left(b_{-1}^{(1)}=c_{-1}^{(1)}=0\right)$ :

$$
(n+1)\left(n+i \eta+\frac{B_{2}}{2}\right) b_{n+1}^{(1)}+\beta_{n}^{(1)} b_{n}^{(1)}+2 i \omega z_{0}\left(n+B_{2}+\frac{B_{1}}{z_{0}}-1\right) b_{n-1}^{(1)}=0
$$

and

$$
\begin{aligned}
& (n+1) c_{n+1}^{(1)}+\beta_{n}^{(1)} c_{n}^{(1)} \\
& +2 i \omega z_{0}\left(n+B_{2}+\frac{B_{1}}{z_{0}}-1\right)\left(n+i \eta+\frac{B_{2}}{2}-1\right) c_{n-1}^{(1)}=0,
\end{aligned}
$$

where

$$
\beta_{n}^{(1)}=n\left(n+B_{2}-1+2 i \omega z_{0}\right)+B_{3}+i \omega z_{0}\left(B_{2}+\frac{B_{1}}{z_{0}}\right) .
$$

According to the previous subsection, if in $+\left(B_{2} / 2\right)=-l(l=0,1,2, \cdots)$, the series in $U_{1}$ and $\bar{U}_{1}^{\infty}$ break off on the right and these solutions reduce to Heun polynomials 
$(0 \leq n \leq l)$, while the solution $U_{1}^{\infty}$ truncates on the left $(n \geq l+1)$. However, there is no need of considering these Heun polynomials since the Barber-Hassé solution (30a) also suplies finite-series solutions with the same characteristic equation.

For the solutions (39a) the series are infinite, with $0 \leq n<\infty$, if

$$
B_{2}+\frac{B_{1}}{z_{0}} \text { and } i \eta+\frac{B_{2}}{2} \text { are not zero or negative integers, }
$$

as we see from the recurrence relations (39b) and (39c). The solutions can be connected by means of Eq. (27) if

$$
B_{2}, \frac{B_{2}}{2}-i \eta \text { and } i \eta+\frac{B_{2}}{2} \text { are not zero or negative integers, }
$$

as we see from conditions (29). Then, the relation $c_{n}^{(1)}=\Gamma\left[n+i \eta+\left(B_{2} / 2\right)\right] b_{n}^{(1)}$ is well defined and Heun polynomials are excluded from (39a). Under the above conditions, the coefficients of the Barber-Hassé solution (30a) are proportional to $c_{n}^{(1)}$, that is,

$$
a_{n}^{(1)}=\frac{c_{n}^{(1)}}{\left(z_{0}\right)^{n} \Gamma\left[n+B_{2}+\left(B_{1} / z_{0}\right)\right]} .
$$

This gives another reason to consider the Barber-Hassé solutions in conjunction with the expansions in confluent hypergeometric functions.

Finally, notice that there is another type of finite series which is not included in the Barber-Hassé expansions. It occurs when

$$
B_{2}+\frac{B_{1}}{z_{0}}=-l, \quad l=1,2,3, \cdots,
$$

in which case the three series in (39a) terminate at $n=l$. These Heun polynomials are important because they are preserved by the Whittaker-Ince limit, in opposition to the Heun polynomials obtained from the Barber-Hassé expansions.

\subsection{Convergence of the third solution}

For one-sided infinite series the convergence of $\bar{U}_{1}^{\infty}$ have already been established in Ref. [16]. Next we show that the two-sided infinite series converges in both directions, that is, when $n \rightarrow \infty$ and when $n \rightarrow-\infty$. First we write the solution as

$$
\bar{U}_{1}^{(\infty)}(z)=e^{i \omega z} \sum_{n=-\infty}^{\infty} c_{n}^{(1)} y^{1-B_{2}-n-\nu} \Psi_{n}(y)
$$

where

$$
\Psi_{n}(y)=\Psi\left(1+i \eta-\frac{B_{2}}{2}, 2-n-\nu-B_{2} ; y\right), \quad y=-2 i \omega z
$$

To determine the convergence of the series, we have to find the ratios

$$
\lim _{n \rightarrow \infty} \frac{c_{n+1}^{(1)} \Psi_{n+1}(y)}{c_{n}^{(1)} y \Psi_{n}(y)}, \quad \lim _{n \rightarrow-\infty} \frac{c_{n-1}^{(1)} y \Psi_{n-1}(y)}{c_{n}^{(1)} \Psi_{n}(y)} .
$$

For this, in the first place we divide the recurrence relations (33c) by $n c_{n}^{(1)}$ and retain only the leading terms, that is,

$$
\begin{aligned}
& {\left[1+\frac{\nu+1}{n}\right] \frac{c_{n+1}^{(1)}}{c_{n}^{(1)}}+\left[n+2 \nu+B_{2}-1+2 i \omega z_{0}+O\left(\frac{1}{n}\right)\right]} \\
& +2 i \omega z_{0}\left[n+2 \nu+\frac{3}{2} B_{2}+\frac{B_{1}}{z_{0}}+i \eta-2+O\left(\frac{1}{n}\right)\right] \frac{c_{n-1}^{(1)}}{c_{n}^{(1)}}=0 .
\end{aligned}
$$


The minimal solutions for this equation are

$$
\begin{aligned}
& \lim _{n \rightarrow+\infty} \frac{c_{n+1}^{(1)}}{c_{n}^{(1)}}=-2 i \omega z_{0}\left[1+\frac{1}{n}\left(i \eta-1+\frac{B_{1}}{z_{0}}+\frac{B_{2}}{2}\right)\right], \\
& \lim _{n \rightarrow-\infty} \frac{c_{n-1}^{(1)}}{c_{n}^{(1)}}=-\frac{1}{n}\left[1-\frac{B_{2}+\nu-3}{n}\right] .
\end{aligned}
$$

On the other hand, from Eqs. (B.4) we get

$$
-\left(n+\nu+i \eta+\frac{B_{2}}{2}\right) \Psi_{n+1}(y)+\left(n+\nu+B_{2}-1-y\right) \Psi_{n}(y)+y \Psi_{n-1}(y)=0 .
$$

Dividing this by $n \Psi_{n}$ we obtain

$$
-\left[1+\frac{1}{n}\left(\nu+i \eta+\frac{B_{2}}{2}\right)\right] \frac{\Psi_{n+1}}{\Psi_{n}}+\left[1+\frac{\nu+B_{2}-1-y}{n}\right]+\frac{y}{n} \frac{\Psi_{n-1}}{\Psi_{n}}=0 .
$$

Then we can verify that

$$
\lim _{n \rightarrow+\infty} \frac{\Psi_{n+1}}{\Psi_{n}}=1-\frac{1}{n}\left(1+i \eta-\frac{B_{2}}{2}\right), \quad \lim _{n \rightarrow-\infty} \frac{\Psi_{n-1}}{\Psi_{n}}=-\frac{1}{y}\left(n+\nu+B_{2}-1\right) .
$$

In fact there are other possibilities, but the preceding are the only ones compatible with Eqs. (B.5) and (B.6), respectively. Hence, since $y=-2 i \omega z$, we find

$$
\begin{aligned}
& \lim _{n \rightarrow \infty} \frac{c_{n+1}^{(1)} \Psi_{n+1}(y)}{c_{n}^{(1)} y \Psi_{n}(y)}=\frac{z_{0}}{z}\left[1+\frac{1}{n}\left(B_{2}+\frac{B_{1}}{z_{0}}-2\right)\right], \\
& \lim _{n \rightarrow-\infty} \frac{c_{n-1}^{(1)} y \Psi_{n-1}(y)}{c_{n}^{(1)} \Psi_{n}(y)}=1+\frac{2}{n} .
\end{aligned}
$$

Therefore, by the ratio test [41] the series in Eq. (43) converges in the region $|z|>\mid z_{0}$.

\subsection{Other sets of solutions for the $\mathrm{CHE}$}

In order to generate other solutions for the CHE we use the transformations rules resulting from substitutions of variables which leave the form of the CHE unaltered but change its parameters. Thus, if $U(z)=U\left(B_{1}, B_{2}, B_{3} ; z_{0}, \omega, \eta ; z\right)$ denotes one solution of the CHE in the Leaver form (3), we have the rules $T_{1}, T_{2}, T_{3}$ and $T_{4}$ which operate as [16]

$$
\begin{array}{ll}
T_{1} U(z)=z^{1+B_{1} / z_{0}} U\left(C_{1}, C_{2}, C_{3} ; z_{0}, \omega, \eta ; z\right), & z_{0} \neq 0, \\
T_{2} U(z)=\left(z-z_{0}\right)^{1-B_{2}-B_{1} / z_{0}} U\left(B_{1}, D_{2}, D_{3} ; z_{0}, \omega, \eta ; z\right), & z_{0} \neq 0, \\
T_{3} U(z)=U\left(B_{1}, B_{2}, B_{3} ; z_{0},-\omega,-\eta ; z\right), & \forall z_{0}, \\
T_{4} U(z)=U\left(-B_{1}-B_{2} z_{0}, B_{2}, B_{3}+2 \eta \omega z_{0} ; z_{0},-\omega, \eta ; z_{0}-z\right), & \forall z_{0},
\end{array}
$$

where

$$
\begin{aligned}
& C_{1}=-B_{1}-2 z_{0}, \quad C_{2}=2+B_{2}+\frac{2 B_{1}}{z_{0}}, C_{3}=B_{3}+\left(1+\frac{B_{1}}{z_{0}}\right)\left(B_{2}+\frac{B_{1}}{z_{0}}\right), \\
& D_{2}=2-B_{2}-\frac{2 B_{1}}{z_{0}}, D_{3}=B_{3}+\frac{B_{1}}{z_{0}}\left(\frac{B_{1}}{z_{0}}+B_{2}-1\right) .
\end{aligned}
$$

Applying these rules to the basic set (33a) we may generate a group containing 16 sets of solutions for the CHE. We will write only the subgroup obtained by using the

rules $T_{1}$ and $T_{2}$ in this order:

$$
\left(U_{1}, U_{1}^{\infty}, \bar{U}_{1}^{\infty}\right) \stackrel{T_{1}}{\longleftrightarrow}\left(U_{2}, U_{2}^{\infty}, \bar{U}_{2}^{\infty}\right) \stackrel{T_{2}}{\longleftrightarrow}\left(U_{3}, U_{3}^{\infty}, \bar{U}_{3}^{\infty}\right) \stackrel{T_{1}}{\longleftrightarrow}\left(U_{4}, U_{4}^{\infty}, \bar{U}_{4}^{\infty}\right)
$$


where $\left(U_{1}, U_{1}^{\infty}, \bar{U}_{1}^{\infty}\right)$ denotes the first set of solutions (33a).

In each of the following sets, the conditions for doubly infinite series are obtained by choosing $\nu$ such that the coefficients of $c_{n}^{(i)}$ and $c_{n-1}^{(i)}$ do not vanish in the recurrence relations. Besides this, one solution can be expressed as a linear combination of the others, if $a, c$ and $c-a$ are not negative integers, where $a$ and $c$ are respectively the first and the second parameters of the confluent hypergeometric functions: these conditions lead to some restrictions on the values of $\nu$ as well as on the parameters of the CHE. Despite the notation, it is understood that the parameter $\nu$ may be different in each set of solutions.

The second set of solutions admits the Whittaker-Ince limit but does not admit the Leaver limit. It reads

$$
\begin{gathered}
U_{2}(z)=e^{-i \omega z} z^{1+\frac{B_{1}}{z_{0}}} \times \\
\sum_{n}(-1)^{n} b_{n}^{(2)} \widetilde{\Phi}\left(1-i \eta+\frac{B_{1}}{z_{0}}+\frac{B_{2}}{2}, n+\nu+2+B_{2}+\frac{2 B_{1}}{z_{0}} ; 2 i \omega z\right), \\
U_{2}^{\infty}(z)=e^{-i \omega z} z^{1+\frac{B_{1}}{z_{0}}} \times \\
\sum_{n}(-1)^{n} b_{n}^{(2)} \Psi\left(1-i \eta+\frac{B_{1}}{z_{0}}+\frac{B_{2}}{2}, n+\nu+2+B_{2}+\frac{2 B_{1}}{z_{0}} ; 2 i \omega z\right), \\
\bar{U}_{2}^{\infty}(z)=e^{i \omega z} z^{1+\frac{B_{1}}{z_{0}} \times} \\
\sum_{n} c_{n}^{(2)} \Psi\left(n+\nu+1+i \eta+\frac{B_{1}}{z_{0}}+\frac{B_{2}}{2}, n+\nu+2+B_{2}+\frac{2 B_{1}}{z_{0}} ;-2 i \omega z\right),
\end{gathered}
$$

where the recurrence relations for $c_{n}^{(2)}$ are

$$
\begin{aligned}
& (n+\nu+1) c_{n+1}^{(2)}+\left[(n+\nu)\left(n+\nu+1+2 i \omega z_{0}+B_{2}+\frac{2 B_{1}}{z_{0}}\right)\right. \\
& \left.+B_{3}+\left(1+\frac{B_{1}}{z_{0}}\right)\left(B_{2}+\frac{B_{1}}{z_{0}}\right)+i \omega z_{0}\left(B_{2}+\frac{B_{1}}{z_{0}}\right)\right] c_{n}^{(2)} \\
& +2 i \omega z_{0}\left(n+\nu+B_{2}+\frac{B_{1}}{z_{0}}-1\right)\left(n+\nu+i \eta+\frac{B_{1}}{z_{0}}+\frac{B_{2}}{2}\right) c_{n-1}^{(2)}=0 .
\end{aligned}
$$

The recurrence relations for $b_{n}^{(2)}$ are obtained from the previous ones by

$$
c_{n}^{(2)}=\Gamma\left[n+\nu+1+i \eta+\frac{B_{1}}{z_{0}}+\frac{B_{2}}{2}\right] b_{n}^{(2)} .
$$

The third set of solutions, which admits both limits, is

$$
\begin{aligned}
U_{3}(z)= & e^{-i \omega z}\left(z-z_{0}\right)^{1-B_{2}-\frac{B_{1}}{z_{0}}} z^{1+\frac{B_{1}}{z_{0}} \times} \\
& \sum_{n}(-1)^{n} b_{n}^{(3)} \widetilde{\Phi}\left(2-i \eta-\frac{B_{2}}{2}, n+\nu+4-B_{2} ; 2 i \omega z\right), \\
U_{3}^{\infty}(z)= & e^{-i \omega z}\left(z-z_{0}\right)^{1-B_{2}-\frac{B_{1}}{z_{0}}} z^{1+\frac{B_{1}}{z_{0}} \times} \\
& \sum_{n}(-1)^{n} b_{n}^{(3)} \Psi\left(2-i \eta-\frac{B_{2}}{2}, n+\nu+4-B_{2} ; 2 i \omega z\right), \\
\bar{U}_{3}^{\infty}(z)= & e^{i \omega z}\left(z-z_{0}\right)^{1-B_{2}-\frac{B_{1}}{z_{0}}} z^{1+\frac{B_{1}}{z_{0}} \times} \\
& \sum_{n} c_{n}^{(3)} \Psi\left(n+\nu+2+i \eta-\frac{B_{2}}{2}, n+\nu+4-B_{2} ;-2 i \omega z\right),
\end{aligned}
$$


where the $c_{n}^{(3)}$ satisfy (CORRIGIR)

$$
\begin{aligned}
& (n+\nu+1) c_{n+1}^{(3)} \\
& +\left[(n+\nu)\left(n+\nu+3-B_{2}+2 i \omega z_{0}\right)+i \omega z_{0}\left(2-B_{2}-\frac{B_{1}}{z_{0}}\right)+B_{3}+2-B_{2}\right] c_{n}^{(3)} \\
& +2 i \omega z_{0}\left(n+\nu+1-B_{2}-\frac{B_{1}}{z_{0}}\right)\left(n+\nu+1+i \eta-\frac{B_{2}}{2}\right) c_{n-1}^{(3)}=0
\end{aligned}
$$

and the recurrence relations for $b_{n}^{(3)}$ follow from

$$
c_{n}^{(3)}=\Gamma\left[n+\nu+i \eta+2-\frac{B_{2}}{2}\right] b_{n}^{(3)} .
$$

The fourth set of solutions is

$$
\begin{gathered}
U_{4}(z)=e^{-i \omega z}\left(z-z_{0}\right)^{1-B_{2}-\frac{B_{1}}{z_{0}} \times} \\
\sum_{n}(-1)^{n} b_{n}^{(4)} \widetilde{\Phi}\left(1-i \eta-\frac{B_{1}}{z_{0}}-\frac{B_{2}}{2}, n+\nu+2-B_{2}-\frac{2 B_{1}}{z_{0}} ; 2 i \omega z\right), \\
U_{4}^{\infty}(z)=e^{-i \omega z}\left(z-z_{0}\right)^{1-B_{2}-\frac{B_{1}}{z_{0}} \times} \\
\sum_{n}(-1)^{n} b_{n}^{(4)} \Psi\left(1-i \eta-\frac{B_{1}}{z_{0}}-\frac{B_{2}}{2}, n+\nu+2-B_{2}-\frac{2 B_{1}}{z_{0}} ; 2 i \omega z\right), \\
\bar{U}_{4}^{\infty}(z)=e^{i \omega z}\left(z-z_{0}\right)^{1-B_{2}-\frac{B_{1}}{z_{0}} \times} \times{ }_{n}^{(4)} \Psi\left(n+\nu+1+i \eta-\frac{B_{1}}{z_{0}}-\frac{B_{2}}{2}, n+\nu+2-B_{2}-\frac{2 B_{1}}{z_{0}} ;-2 i \omega z\right),
\end{gathered}
$$

where the recurrence relations for $c_{n}^{(4)}$ are

$$
\begin{aligned}
& (n+\nu+1) c_{n+1}^{(4)}+\left[(n+\nu)\left(n+\nu+1-B_{2}-\frac{2 B_{1}}{z_{0}}+2 i \omega z_{0}\right)\right. \\
& \left.+B_{3}+\frac{B_{1}}{z_{0}}\left(B_{2}+\frac{B_{1}}{z_{0}}-1\right)+i \omega z_{0}\left(2-B_{2}-\frac{B_{1}}{z_{0}}\right)\right] c_{n}^{(4)} \\
& +2 i \omega z_{0}\left(n+\nu+1-B_{2}-\frac{B_{1}}{z_{0}}\right)\left(n+\nu+i \eta-\frac{B_{2}}{2}-\frac{B_{1}}{z_{0}}\right) c_{n-1}^{(4)}=0 .
\end{aligned}
$$

The $b_{n}^{(4)}$ and $c_{n}^{(4)}$ are connected by

$$
c_{n}^{(4)}=\Gamma\left[n+\nu+i \eta+1-\frac{B_{2}}{2}-\frac{B_{1}}{z_{0}}\right] b_{n}^{(4)} .
$$

One-sided infinite series result when we take $\nu=0$ in the two-sided series solutions, since this restricts the summation to $n \geq 0$.

\section{Whittaker-Ince Limit of the Confluent Heun Equation}

In this section we show that for the Whittaker-Ince limit of the CHE, that is, for equation

$$
z\left(z-z_{0}\right) \frac{d^{2} U}{d z^{2}}+\left(B_{1}+B_{2} z\right) \frac{d U}{d z}+\left[B_{3}+q\left(z-z_{0}\right)\right] U=0, \quad(q \neq 0),
$$

the solutions of the $\mathrm{CHE}$ reduce to expansions in series of Bessel functions. The procedure is the same used in [16] for one-sided solutions, but we correct the following systematic error: the Bessel functions of the first kind $J_{\lambda}$ which appear in the solutions of Ref. [16] must be replaced by $(-1)^{n} J_{\lambda}$. 
In Sec. 3.1 we write the first set of solutions for Eq. (53). We find that the three solutions have exactly the same series coefficients and no restriction must be imposed on the parameters of Eq. (53) in order to write one solution as a linear combination of the others. In Sec. 3.2 we show how these solutions are obtained from the solutions of the CHE by using the Whittaker-Ince limit (5). In Sect. 3.3 we use the transformations rules for Eq. (53) to generate other sets of solutions. The limits of the Barber-Hassé solutions are given in [16], but such expansions are unsuitable to get finite-series solutions.

\subsection{The first set of solutions}

The Whittaker-Ince limit of the fundamental set of solutions given in Eqs. (33a) yields $(i=1,2)$

$$
\begin{aligned}
& U_{1}(z)=\sum_{n}(-1)^{n} c_{n}^{(1)}(\sqrt{q z})^{-\left(n+\nu+B_{2}-1\right)} J_{n+\nu+B_{2}-1}(2 \sqrt{q z}), \quad \forall z, \\
& U_{1}^{(i)}(z)=\sum_{n}(-1)^{n} c_{n}^{(1)}(\sqrt{q z})^{-\left(n+\nu+B_{2}-1\right)} H_{n+\nu+B_{2}-1}^{(i)}(2 \sqrt{q z}), \quad|z|>\left|z_{0}\right|,
\end{aligned}
$$

where the limits of the recurrence relations (33b) and (33c) are

$$
\begin{aligned}
& (n+\nu+1) c_{n+1}^{(1)}+\left[(n+\nu)\left(n+\nu+B_{2}-1\right)+B_{3}\right] c_{n}^{(1)} \\
& +q z_{0}\left[n+\nu+B_{2}+\left(B_{1} / z_{0}\right)-1\right] c_{n-1}^{(1)}=0 .
\end{aligned}
$$

In these solutions $J_{\lambda}(x)$ denotes Bessel functions of the first kind of order $\lambda$, whereas $H_{\lambda}^{(1)}(x)$ and $H_{\lambda}^{(2)}(x)$ denote Hankel functions of first and second kind respectively. The solution $U_{1}(z)$ comes from the solution $U_{1}(z)$ of the CHE, while the solutions denoted by $U_{i}^{(i)}(z)$ follow either from $U_{1}^{(\infty)}(z)$ or $\bar{U}_{1}^{(\infty)}(z)$. This set admits the Leaver limit $z_{0} \rightarrow 0$.

The Bessel functions which appear in the solutions are all independent since their Wronskians are 13

$$
\begin{aligned}
& W\left[J_{\lambda}(x), H_{\lambda}^{(1)}(x)\right]=2 i /(\pi x), \quad W\left[J_{\lambda}(x), H_{\lambda}^{(2)}(x)\right]=-2 i /(\pi x), \\
& W\left[H_{\lambda}^{(1)}(x), H_{\lambda}^{(2)}(x)\right]=-4 i /(\pi x) .
\end{aligned}
$$

Then, the relation

$$
J_{\lambda}(x)=\frac{1}{2}\left[H_{\lambda}^{(1)}(x)+H_{\lambda}^{(2)}(x)\right]
$$

can be used to write each solution as a linear combination of the others in a region where the three solutions are valid.

On the other hand, for a fixed $\lambda$ the asymptotic behaviours of the Bessel functions as $|x| \rightarrow \infty$ are 13

$$
\begin{array}{ll}
J_{\lambda}(x) \sim \sqrt{\frac{2}{\pi x}} \cos \left(x-\frac{1}{2} \lambda \pi-\frac{1}{4} \pi\right), & |\arg x|<\pi ; \\
H_{\lambda}^{(1)}(x) \sim \sqrt{2 /(\pi x)} e^{i\left(x-\frac{1}{2} \lambda \pi-\frac{1}{4} \pi\right)}, & -\pi<\arg x<2 \pi ; \\
H_{\lambda}^{(2)}(x) \sim \sqrt{2 /(\pi x)} e^{-i\left(x-\frac{1}{2} \lambda \pi-\frac{1}{4} \pi\right)}, & -2 \pi<\arg x<\pi .
\end{array}
$$

Thus, for the solutions $U_{1}^{(i)}$ we find

$$
\lim _{z \rightarrow \infty} U_{1}^{(1)}(z) \sim e^{2 i \sqrt{q z}} z^{\frac{1}{4}-\frac{B_{2}}{2}-\frac{\nu}{2}}, \quad \lim _{z \rightarrow \infty} U_{1}^{(2)}(z) \sim e^{-2 i \sqrt{q z}} z^{\frac{1}{4}-\frac{B_{2}}{2}-\frac{\nu}{2}}
$$


The behaviour of $U_{1}$ when $z \rightarrow \infty$ is a linear combination of these due to Eq. (55).

Notice that, by setting

$$
\begin{aligned}
& w(u)=U(z), \quad z=\cos ^{2}(\sigma u), \quad(\sigma=1, i), \\
& z_{0}=1, \quad B_{1}=-\frac{1}{2}, \quad B_{2}=1, \quad B_{3}=\frac{k^{2}}{2}-\frac{a}{4}, \quad q=k^{2},
\end{aligned}
$$

in Eq. (53), we obtain the Mathieu equation (12). Then, from the solutions (54a) and (54b) we get the following even solutions for the Mathieu equation

$$
\begin{aligned}
& w_{1}(u)=\sum_{n}(-1)^{n} c_{n}^{(1)}[k \cos (\sigma u)]^{-n-\nu} J_{n+\nu}(2 k \cos (\sigma u)), \quad \forall u, \\
& w_{1}^{(i)}(u)=\sum_{n}(-1)^{n} c_{n}^{(1)}[k \cos (\sigma u)]^{-n-\nu} H_{n+\nu}^{(i)}(2 k \cos (\sigma u)), \quad|\cos (\sigma u)|>1,
\end{aligned}
$$

where the coefficients $c_{n}^{(1)}$ satisfy

$$
(n+\nu+1) c_{n+1}^{(1)}+\left[(n+\nu)^{2}+\left(k^{2} / 2\right)-(a / 4)\right] c_{n}^{(1)}+k^{2}[n+\nu-(1 / 2)] c_{n-1}^{(1)}=0 .
$$

In this set of two-sided infinity series solutions, the first solution converges for any $u$, in contrast with the usual two-sided solutions for the Mathieu equation which converge, all of them, only for $|\cos (\sigma u)|>1$ [1, 32.

One-sided infinite series are obtained by putting $\nu=0$ in the two-sided series solutions, what restricts the summation to $n \geq 0$.

\subsection{Derivation of the solutions}

To compute the Whittaker-Ince limits, first we rewrite the solutions of the CHE in a form convenient for using the formulas (B.7) and (B.8). Thus, we rewrite the solutions (33a) as $(q=-2 \eta \omega)$

$$
\begin{aligned}
& U_{1}(z)=e^{-i \omega z} \sum_{n} \frac{(-1)^{n} c_{n}^{(1)}}{\Gamma\left(n+\nu+B_{2}\right)} \Phi\left(\frac{B_{2}}{2}-i \eta, n+\nu+B_{2} ; \frac{q z}{i \eta}\right) \\
& U_{1}^{\infty}(z)=e^{-i \omega z} \sum_{n} D_{n} \Gamma\left(1-i \eta-n-\nu-\frac{B_{2}}{2}\right) \Psi\left(\frac{B_{2}}{2}-i \eta, n+\nu+B_{2} ; \frac{q z}{i \eta}\right) \\
& \bar{U}_{1}^{\infty}(z)=e^{i \omega z} \sum_{n} E_{n}(-q z)^{1-n-\nu-B_{2}} \Gamma\left(i \eta+n+\nu+\frac{B_{2}}{2}\right) \times \\
& \Psi\left(1+i \eta-\frac{B_{2}}{2}, 2-n-\nu-B_{2} ;-\frac{q z}{i \eta}\right)
\end{aligned}
$$

where the new coefficients are defined by

$$
D_{n}=\frac{(-1)^{n} b_{n}^{(1)}}{\Gamma\left[1-n-\nu-i \eta-\left(B_{2} / 2\right)\right]}, \quad E_{n}=\frac{(i \eta)^{n+\nu} c_{n}^{(1)}}{\Gamma\left[i \eta+n+\nu+\left(B_{2} / 2\right)\right]} .
$$

Inserting these relations into Eqs. (33b) and (33c) we find

$$
\begin{aligned}
& (n+\nu+1) D_{n+1}+\beta_{n}^{(1)} D_{n} \\
& +2 i \omega z_{0}\left[n+\nu+i \eta+\left(B_{2} / 2\right)-1\right]\left[n+\nu+B_{2}+\left(B_{1} / z_{0}\right)-1\right] D_{n-1}=0,
\end{aligned}
$$

and

$$
\begin{aligned}
& (n+\nu+1)\left[\frac{n+\nu+i \eta+\left(B_{2} / 2\right)}{i \eta}\right] E_{n+1}+\beta_{n}^{(1)} E_{n} \\
& -2 \eta \omega z_{0}\left[n+\nu+B_{2}+\left(B_{1} / z_{0}\right)-1\right] E_{n-1}=0,
\end{aligned}
$$


where

$$
\beta_{n}^{(1)}=(n+\nu)\left(n+\nu+B_{2}-1+2 i \omega z_{0}\right)+B_{3}+i \omega z_{0}\left(B_{2}+\frac{B_{1}}{z_{0}}\right) .
$$

Thence, we find that the Whittaker-Ince limits of these relations are identical since

$$
\lim c_{n}^{(1)}=\lim D_{n}=\lim E_{n}, \quad(\omega \rightarrow 0, \quad \eta \rightarrow \infty, \quad 2 \eta \omega=-q) .
$$

Denoting by $c_{n}^{(1)}$ the above limits, we obtain the recurrence relations (54b).

Now, by letting $(-i \eta) \rightarrow \infty$ and using Eq. (B.7) we find

$$
\Phi\left(\frac{B_{2}}{2}-i \eta, n+\nu+B_{2} ; \frac{q z}{i \eta}\right) \rightarrow \Gamma\left(n+\nu+B_{2}\right)(\sqrt{q z})^{1-n-\nu-B_{2}} J_{n+\nu+B_{2}-1}(2 \sqrt{q z}) .
$$

Thus the limit of Eq. (59a) is the solution $U_{1}(z)$ written in Eqs. (54a). On the other hand, to obtain the limit of the solution (59b) we define $L_{1}(z)$ by

$$
L_{1}(z):=\Gamma\left(1-i \eta-n-\nu-\frac{B_{2}}{2}\right) \Psi\left(\frac{B_{2}}{2}-i \eta, n+\nu+B_{2} ; \frac{q z}{i \eta}\right) .
$$

Then, when $(-i \eta) \rightarrow \infty$ the relation (B.8) gives

$$
\begin{aligned}
& L_{1}(z) \rightarrow 2( \pm i \sqrt{q z})^{1-n-\nu-B_{2}} K_{n+\nu+B_{2}-1}( \pm 2 i \sqrt{q z})= \\
& \left\{\begin{array}{c}
-i \pi e^{i \pi\left(1-n-\nu-B_{2}\right)}(\sqrt{q z})^{1-n-\nu-B_{2}} H_{n+\nu+B_{2}-1}^{(2)}(2 \sqrt{q z}), \\
i \pi e^{-i \pi\left(1-n-\nu-B_{2}\right)}(\sqrt{q z})^{1-n-\nu-B_{2}} H_{n+\nu+B_{2}-1}^{(1)}(2 \sqrt{q z}),
\end{array}\right.
\end{aligned}
$$

where in last step we have used the relations among the functions $K_{\lambda}$ and $H_{\lambda}^{(i)}$ given in Eq. (B.10). Inserting these into the limit of the solution (59b) and supressing multiplicative factors, we find the solutions $U_{1}^{(i)}(z)$ given in (54a). The same solutions follow from the limit of the solution (59c). Notice that these are formal derivations which involve some tricks. However, we may check the resulting solutions by inserting them into Eq. (53).

\subsection{Other sets of solutions for Whittaker-Ince Limit of the CHE}

If $U(z)=U\left(B_{1}, B_{2}, B_{3} ; z_{0}, q ; z\right)$ denotes one solution (or set of solutions) for Whittaker-Ince limit (6) of the CHE, then the rules $\mathscr{T}_{1}, \mathscr{T}_{2}$ and $\mathscr{T}_{3}$ given by [15]

$$
\begin{aligned}
& \mathscr{T}_{1} U(z)=z^{1+B_{1} / z_{0}} U\left(C_{1}, C_{2}, C_{3} ; z_{0}, q ; z\right), \quad z_{0} \neq 0, \\
& \mathscr{T}_{2} U(z)=\left(z-z_{0}\right)^{1-B_{2}-B_{1} / z_{0}} U\left(B_{1}, D_{2}, D_{3} ; z_{0}, q ; z\right), \quad z_{0} \neq 0, \\
& \mathscr{T}_{3} U(z)=U\left(-B_{1}-B_{2} z_{0}, B_{2}, B_{3}-q z_{0} ; z_{0},-q ; z_{0}-z\right), \quad \forall z_{0}
\end{aligned}
$$

can generate a group having 8 solutions. The constants $C_{i}$ and $D_{i}$ are defined in Eqs. (48b). Next we use only $\mathscr{T}_{1}$ and $\mathscr{T}_{2}$, following the sequence

$$
\left(U_{1}, U_{1}^{(i)}\right) \stackrel{\mathscr{T}_{1}}{\longleftrightarrow}\left(U_{2}, U_{2}^{(i)}\right) \stackrel{\mathscr{T}_{2}}{\longleftrightarrow}\left(U_{3}, U_{3}^{(i)}\right) \stackrel{\mathscr{T}_{1}}{\longleftrightarrow}\left(U_{4}, U_{4}^{(i)}\right)
$$

where $\left(U_{1}, U_{1}^{(i)}\right)$ denotes the set of solutions written in Eqs. (54a) and (54b). The conditions for having doubly infinite series are obtained by choosing $\nu$ such that the coefficients of $c_{n}^{(i)}$ and $c_{n-1}^{(i)}$ do not vanish in the recurrence relations, as in the case of the CHE. Besides this, according to Eq. (55) one solution can be expressed as a linear combination of the other solutions. We will write only the solutions in series of Bessel functions of the first kind, $J_{\lambda}$; the others are obtained by replacing $J_{\lambda}$ by 
the first and second Hankel functions $H_{\lambda}^{(i)}(i=1,2)$. The solutions for the Mathieu equation are derived by using Eq. (57).

The first solution of the second set, which does not admit the Leaver limit $z_{0} \rightarrow 0$, is $(\forall z)$

$$
U_{2}(z)=z^{1+\frac{B_{1}}{z_{0}}} \sum_{n}(-1)^{n} c_{n}^{(2)}(\sqrt{q z})^{-n-\nu-1-B_{2}-\frac{2 B_{1}}{z_{0}}} J_{n+\nu+1+B_{2}+\frac{2 B_{1}}{z_{0}}}(2 \sqrt{q z}),
$$

where the coefficients satisfy

$$
\begin{aligned}
& (n+\nu+1) c_{n+1}^{(2)}+\left[(n+\nu)\left(n+\nu+B_{2}+1+\frac{2 B_{1}}{z_{0}}\right)+B_{3}\right. \\
& \left.+\left(1+\frac{B_{1}}{z_{0}}\right)\left(B_{2}+\frac{B_{1}}{z_{0}}\right)\right] c_{n}^{(2)}+q z_{0}\left(n+\nu+B_{2}+\frac{B_{1}}{z_{0}}-1\right) c_{n-1}^{(2)}=0 .
\end{aligned}
$$

For the Mathieu equation the corresponding solutions are even, as those of the first set, since

$$
w_{2}(u)=\cos (\sigma u) \sum_{n}(-1)^{n} c_{n}^{(2)}[k \cos (\sigma u)]^{-n-\nu-1} J_{n+\nu+1}(2 k \cos (\sigma u)), \quad \forall u,
$$

with

$$
\begin{aligned}
& (n+\nu+1) c_{n+1}^{(2)}+\left[(n+\nu)(n+\nu+1)+\frac{k^{2}}{2}+\frac{1-a}{4}\right] c_{n}^{(2)} \\
& +k^{2}[n+\nu-(1 / 2)] c_{n-1}^{(2)}=0 .
\end{aligned}
$$

In third set, which admits the Leaver limit $z_{0} \rightarrow 0$, the first solution is

$$
\begin{aligned}
U_{3}(z)= & z^{1+\frac{B_{1}}{z_{0}}}\left(z-z_{0}\right)^{1-B_{2}-\frac{B_{1}}{z_{0}} \times} \\
& \sum_{n}(-1)^{n} c_{n}^{(3)}(\sqrt{q z})^{-n-\nu-3+B_{2}} J_{n+\nu+3-B_{2}}(2 \sqrt{q z}), \quad \forall z
\end{aligned}
$$

associated with the recurrence relations

$$
\begin{aligned}
& (n+\nu+1) c_{n+1}^{(3)}+\left[(n+\nu)\left(n+\nu+3-B_{2}\right)+B_{3}+2-B_{2}\right] c_{n}^{(3)} \\
& +q z_{0}\left[n+\nu+1-B_{2}-\left(B_{1} / z_{0}\right)\right] c_{n-1}^{(3)}=0 .
\end{aligned}
$$

For the Mathieu equation, this gives the odd solution:

$$
w_{3}(u)=\sin (2 \sigma u) \sum_{n}(-1)^{n} c_{n}^{(3)}[k \cos (\sigma u)]^{-n-\nu-2} J_{n+\nu+2}(2 k \cos (\sigma u)), \quad \forall u,
$$

where

$$
\begin{aligned}
& (n+\nu+1) c_{n+1}^{(3)}+\left[(n+\nu)(n+\nu+2)+\frac{k^{2}}{2}-\frac{a}{4}+1\right] c_{n}^{(3)} \\
& +k^{2}[n+\nu+(1 / 2)] c_{n-1}^{(3)}=0 .
\end{aligned}
$$

The fourth set of solutions, which does not admit the Leaver limit, gives

$$
\begin{aligned}
& U_{4}(z)=\left(z-z_{0}\right)^{1-B_{2}-\frac{B_{1}}{z_{0}}} \times \\
& \quad \sum_{n}(-1)^{n} c_{n}^{(4)}(\sqrt{q z})^{-n-\nu-1+B_{2}+\frac{2 B_{1}}{z_{0}}} J_{n+\nu+1-B_{2}-\frac{2 B_{1}}{z_{0}}}(2 \sqrt{q z}), \quad \forall z,
\end{aligned}
$$


with the recurrence relations

$$
\begin{aligned}
& (n+\nu+1) c_{n+1}^{(4)}+\left[(n+\nu)\left(n+\nu+1-B_{2}-\frac{2 B_{1}}{z_{0}}\right)+B_{3}\right. \\
& \left.+\frac{B_{1}}{z_{0}}\left(B_{2}+\frac{B_{1}}{z_{0}}-1\right)\right] c_{n}^{(4)}+q z_{0}\left(n+\nu+1-B_{2}-\frac{B_{1}}{z_{0}}\right) c_{n-1}^{(4)}=0 .
\end{aligned}
$$

For the Mathieu equation, we find odd solutions:

$$
w_{4}(u)=\sin (\sigma u) \sum_{n}(-1)^{n} c_{n}^{(4)}[k \cos (\sigma u)]^{-n-\nu-1} J_{n+\nu+1}(2 k \cos (\sigma u)), \quad \forall u,
$$

with the recurrence relations

$$
\begin{aligned}
& (n+\nu+1) c_{n+1}^{(4)}+\left[(n+\nu)(n+\nu+1)+\frac{k^{2}}{2}-\frac{a-1}{4}\right] c_{n}^{(4)} \\
& +k^{2}[n+\nu+(1 / 2)] c_{n-1}^{(4)}=0 .
\end{aligned}
$$

\section{The Double-Confluent Heun Equation (DCHE)}

In Sec. 4.1 we obtain sets of solutions for the double-confluent Heun equation (3) by applying the Leaver limit $\left(z_{0} \rightarrow 0\right)$ to solutions of the CHE. The expansions in series of regular hypergeometric functions converge for any $z$, whereas the expansions in series of irregular functions converge for $|z|>0$. We also give the conditions to express one solution in terms of the others and obtain one-sided series solutions by truncating the two-sided series on the left. Finally, in Sec. 4.2 we write the solutions for Eq. (17), that is, for the Whittaker-Ince limit of the DCHE.

\subsection{Solutions for the general case}

For $z_{0} \rightarrow 0$ the solutions (33a) are not affected formally, but their recurrence relations change. We find

$$
\begin{aligned}
& U_{1}(z)=e^{-i \omega z} \sum_{n}(-1)^{n} b_{n}^{(1)} \widetilde{\Phi}\left(\frac{B_{2}}{2}-i \eta, n+\nu+B_{2} ; 2 i \omega z\right) \\
& U_{1}^{\infty}(z)=e^{-i \omega z} \sum_{n}(-1)^{n} b_{n}^{(1)} \Psi\left(\frac{B_{2}}{2}-i \eta, n+\nu+B_{2} ; 2 i \omega z\right), \\
& \bar{U}_{1}^{\infty}(z)=e^{i \omega z} \sum_{n} c_{n}^{(1)} \Psi\left(n+\nu+i \eta+\frac{B_{2}}{2}, n+\nu+B_{2} ;-2 i \omega z\right) .
\end{aligned}
$$

The recurrence relations for the $c_{n}^{(1)}$ are

$$
\begin{aligned}
& (n+\nu+1) c_{n+1}^{(1)}+\left[(n+\nu)\left(n+\nu+B_{2}-1\right)+i \omega B_{1}+B_{3}\right] c_{n}^{(1)} \\
& +2 i \omega B_{1}\left[n+\nu+i \eta+\left(B_{2} / 2\right)-1\right] c_{n-1}^{(1)}=0
\end{aligned}
$$

wherefrom we get relations for $b_{n}^{(1)}$ by means of

$$
c_{n}^{(1)}=\Gamma\left(n+\nu+i \eta+\frac{B_{2}}{2}\right) b_{n}^{(1)} .
$$

The behaviour given in Eq. (36) for the CHE is also valid for the present solutions.

These three solutions are given by doubly infinite series if

$$
\nu \text { and } \nu+i \eta+\frac{B_{2}}{2} \text { are not integers. }
$$


If, besides this,

$$
\nu+B_{2} \text { is not integer, and } \frac{B_{2}}{2}-i \eta \text { is not zero or negative integer, }
$$

then any of these solutions can be written as a linear combination of the others by using Eq. (27). Again, as in the CHE, only the restriction on $\left(B_{2} / 2\right)-i \eta$ cannot be satisfied by an suitable choice of $\nu$. In addition, if $\left(B_{2} / 2\right)-i \eta=-m(m=0,1,2, \cdots)$, the expansion $U_{1}$ becomes meaningless. For this case, instead of $U_{1}$, we can use

$$
U_{1}^{\mathrm{p}}(z)=e^{-i \omega z} \sum_{n=0}^{\infty} d_{n}^{(1)}\left(\frac{z}{B_{1}}\right)^{n}, \quad(|z|=\text { finite })
$$

where the recurrence relations for the coefficients are $\left(d_{-1}^{(1)}=0\right)$

$$
\begin{aligned}
& (n+1) d_{n+1}^{(1)}+\left[n\left(n+B_{2}-1\right)-i \omega B_{1}+B_{3}\right] d_{n}^{(1)} \\
& -2 i \omega B_{1}\left[n-i \eta+\left(B_{2} / 2\right)-1\right] d_{n-1}^{(1)}=0 .
\end{aligned}
$$

This $U_{1}^{\mathrm{p}}$, which reduces to a Heun polynomial when $\left(B_{2} / 2\right)-i \eta=-m$, was obtained by letting $z_{0} \rightarrow 0$ in Eq. (38a).

A second set follows from the solutions (51a) and the corresponding recurrence relations (51b). Using the L'Hospital rule we find that

$$
z^{1+\frac{B_{1}}{z_{0}}}\left(z-z_{0}\right)^{1-B_{2}-\frac{B_{1}}{z_{0}}}=z\left(z-z_{0}\right)^{1-B_{2}}\left(1-\frac{z_{0}}{z}\right)^{-\frac{B_{1}}{z_{0}}} \rightarrow z^{2-B_{2}} e^{B_{1} / z},\left(z_{0} \rightarrow 0\right)
$$

and, hence, we obtain the solutions

$$
\begin{aligned}
U_{2}(z)= & z^{2-B_{2}} e^{-i \omega z+\frac{B_{1}}{z}} \sum_{n}(-1)^{n} b_{n}^{(2)} \widetilde{\Phi}\left(2-i \eta-\frac{B_{2}}{2}, n+\nu+4-B_{2} ; 2 i \omega z\right), \\
U_{2}^{\infty}(z)= & z^{2-B_{2}} e^{-i \omega z+\frac{B_{1}}{z}} \sum_{n}(-1)^{n} b_{n}^{(2)} \Psi\left(2-i \eta-\frac{B_{2}}{2}, n+\nu+4-B_{2} ; 2 i \omega z\right), \\
\bar{U}_{2}^{\infty}(z)= & z^{2-B_{2}} e^{i \omega z+\frac{B_{1}}{z}} \times \\
& \sum_{n} c_{n}^{(2)} \Psi\left(n+\nu+2+i \eta-\frac{B_{2}}{2}, n+\nu+4-B_{2} ;-2 i \omega z\right),
\end{aligned}
$$

where the coefficients $c_{n}^{(2)}$ obey

$$
\begin{aligned}
& (n+\nu+1) c_{n+1}^{(2)}+\left[(n+\nu)\left(n+\nu+3-B_{2}\right)+B_{3}+2-B_{2}-i \omega B_{1}\right] c_{n}^{(2)} \\
& -2 i \omega B_{1}\left[n+\nu+i \eta+1-\left(B_{2} / 2\right)\right] c_{n-1}^{(2)}=0 .
\end{aligned}
$$

The recurrence relations for the $b_{n}^{(2)}$ are derived from these via the relation

$$
c_{n}^{(2)}=\Gamma\left[n+\nu+i \eta+2-\left(B_{2} / 2\right)\right] b_{n}^{(2)} .
$$

Now the conditions to assure that the three solutions are given by doubly infinite series are

$$
\nu \text { and } \nu+i \eta-\frac{B_{2}}{2} \text { cannot be integers, }
$$

and if, besides this,

$$
\nu+B_{2} \text { is not integer, and } i \eta+\frac{B_{2}}{2} \text { is not zero or negative integer, }
$$


then Eq. (27) can be used to express one solution in terms of the others. If i + $\left(B_{2} / 2\right)=2,3, \cdots$, instead of $U_{2}$ we can use the solution obtained from (75) by the substitution $(\eta, \omega) \rightarrow(-\eta,-\omega)$.

One-sided series solutions are obtained by setting $\nu=0$ in the two-sided solutions, in the same way as in Sect. 2.2. We write only the first set and the limit of the Barber-Hassé solution (30a).

$$
\begin{aligned}
& U_{1}(z)=e^{-i \omega z} \sum_{n=0}^{\infty} \frac{(-1)^{n} c_{n}^{(1)}}{\Gamma\left(n+B_{2}\right)} \Phi\left(\frac{B_{2}}{2}-i \eta, n+B_{2} ; 2 i \omega z\right), \\
& U_{1}^{\infty}(z)=e^{-i \omega z} \sum_{n=0}^{\infty}(-1)^{n} b_{n}^{(1)} \Psi\left(\frac{B_{2}}{2}-i \eta, n+B_{2} ; 2 i \omega z\right), \\
& \bar{U}_{1}^{\infty}(z)=e^{i \omega z} \sum_{n=0}^{\infty} c_{n}^{(1)} \Psi\left(n+i \eta+\frac{B_{2}}{2}, n+B_{2} ;-2 i \omega z\right),
\end{aligned}
$$

where the recurrence relations for $b_{n}^{(1)}$ and $c_{n}^{(1)}$ are $\left(b_{-1}^{(1)}=c_{-1}^{(1)}=0\right)$

$$
\begin{aligned}
& (n+1)\left[n+i \eta+\left(B_{2} / 2\right)\right] b_{n+1}^{(1)}+\left[n\left(n+B_{2}-1\right)+i \omega B_{1}+B_{3}\right] b_{n}^{(1)} \\
& +2 i \omega B_{1} b_{n-1}^{(1)}=0 \\
& \quad(n+1) c_{n+1}^{(1)}+\left[n\left(n+B_{2}-1\right)+i \omega B_{1}+B_{3}\right] c_{n}^{(1)} \\
& +2 i \omega B_{1}\left[n+i \eta+\left(B_{2} / 2\right)-1\right] c_{n-1}^{(1)}=0 .
\end{aligned}
$$

The limit of the Barber-Hassé solution (30a) is

$$
U_{1}^{\text {barber }}(z)=e^{i \omega z} \sum_{n=0}^{\infty} c_{n}^{(1)}\left(\frac{z}{B_{1}}\right)^{n} .
$$

Notice that the solution $U_{1}^{\infty}(z)$ does not admit finite-series solutions, while the other solutions do. However, for finite-series it is sufficient to consider only Barber-Hassé solution. On the other hand, the condition in order that the three expansions in series of confluent hypergeometric functions are given by infinite series $(n \geq 0)$ is

$$
i \eta+\frac{B_{2}}{2} \text { is not zero or negative integer, }
$$

since in this case the series do not truncate on the left or on right. In addition, if

$$
B_{2} \text { and } \frac{B_{2}}{2}-i \eta \text { are not zero or negative integer, }
$$

then Eq. (27) can be used to connect the three one-sided infinite-series solutions.

The Barber-Hassé solution corresponding to the second set is [16]

$$
U_{2}^{\text {barber }}(z)=e^{i \omega z+\left(B_{1} / z\right)} z^{2-B_{2}} \sum_{n=0}^{\infty} c_{n}^{(2)}\left(-\frac{z}{B_{1}}\right)^{n},
$$

where the recurrence relations for $c_{n}^{(2)}$ are obtained by putting $\nu=0$ in Eq. (69b). The corresponding solutions in series of hypergeometric functions can be obtained by taking $\nu=0$ in the solutions (69a). 


\subsection{Solutions for the Whittaker-Ince limit of the DCHE}

For the Whittaker-Ince limit (7) of DCHE, namely,

$$
z^{2} \frac{d^{2} U}{d z^{2}}+\left(B_{1}+B_{2} z\right) \frac{d U}{d z}+\left(B_{3}+q z\right) U=0, \quad\left(q \neq 0, B_{1} \neq 0\right)
$$

there is no finite-series solutions. For this reason, the equation is irrelevant for quasiexactly solvable problems. However, its solutions may be important for studying the scattering of ions which induce dipole and quadrupole moments in polarisable targets [15. Solutions for the above equation can be found by applying either the Whittaker-Ince limit to the solutions given in Sec. 4.1 or the Leaver limit to the solutions (54a) and (63a). We use the latter approach because it is the easiest.

In effect the Leaver limit $z_{0} \rightarrow 0$ does not modify the solutions (54a), which again read

$$
\begin{aligned}
& U_{1}(z)=\sum_{n}(-1)^{n} c_{n}^{(1)}(\sqrt{q z})^{-\left(n+\nu+B_{2}-1\right)} J_{n+\nu+B_{2}-1}(2 \sqrt{q z}), \quad \forall z, \\
& U_{1}^{(i)}(z)=\sum_{n}(-1)^{n} c_{n}^{(1)}(\sqrt{q z})^{-\left(n+\nu+B_{2}-1\right)} H_{n+\nu+B_{2}-1}^{(i)}(2 \sqrt{q z}), \quad|z|>0,
\end{aligned}
$$

but changes their recurrence relations to

$$
(n+\nu+1) c_{n+1}^{(1)}+\left[(n+\nu)\left(n+\nu+B_{2}-1\right)+B_{3}\right] c_{n}^{(1)}+q B_{1} c_{n-1}^{(1)}=0 .
$$

These three solutions are really doubly infinite if $\nu$ is not integer. Furthermore, each solution can be expressed as a linear combination of the others by means of the relation (55).

On the other side, the only detail to get the Leaver limit of the solutions given in Eqs. (63a) is the use the limit written in Eq. (68). The results are

$$
\begin{aligned}
& U_{2}(z)=e^{\frac{B_{1}}{z}} z^{2-B_{2}} \sum_{n}(-1)^{n} c_{n}^{(2)}(\sqrt{q z})^{-n-\nu-3+B_{2}} J_{n+\nu+3-B_{2}}(2 \sqrt{q z}), \quad \forall z ; \\
& U_{2}^{(i)}(z)=e^{\frac{B_{1}}{z}} z^{2-B_{2}} \sum_{n}(-1)^{n} c_{n}^{(2)}(\sqrt{q z})^{-n-\nu-3+B_{2}} H_{n+\nu+3-B_{2}}^{(i)}(2 \sqrt{q z}),|z|>0
\end{aligned}
$$

where

$$
n+\nu+1) c_{n+1}^{(2)}+\left[(n+\nu)\left(n+\nu+3-B_{2}\right)+B_{3}+2-B_{2}\right] c_{n}^{(2)}-q B_{1} c_{n-1}^{(2)}=0 .
$$

One-sided series are obtained by taking $\nu=0$ once more.

\section{Conclusions}

In this section we collect some results we have found, mention other solutions which deserve further analysis, and indicate the possibility of applying the solutions of the four equations given in the first section to physical problems.

We have started with a set of solutions for the confluent Heun equation (CHE) given by three series of confluent hypergeometric functions and, by means of Leaver and the Whittaker-Ince limits, we have derived sets of solutions for all the equations included in the diagram described in the first section. The Barber-Hassé expansions in power series also admit the both limits and can be used to provide solutions for the cases in which the solutions in hypergeometric functions are not valid, as well as to get finite-series solutions for the confluent and double-confluent Heun equations.

In the fundamental set of two-sided solutions $\left(U_{1}, U_{1}^{\infty}, \bar{U}_{1}^{\infty}\right)$, the first and the second solutions are, respectively, Leaver's expansions in series of regular and irregular 
confluent hypergeometric functions for the CHE [25], while $\bar{U}_{1}^{\infty}$ is the two-sided version of the expansion in series of irregular functions given in Ref. [16]. We have seen that, although the solution $U_{1}$ is not valid if there is a certain constraint between two parameters of the equation, we can use the transformation rules to find a two-sided solution valid for that case. We have also established the conditions to express each of the three solutions as a linear combination of the others.

The fact that the two-sided expansion $U_{1}$ in series of regular functions converges for any $z$, and in particular in a neighbourhood of $z=0$, distinguishes the present solutions from the Leaver expansions in series of Coulomb wave functions [25], since the latter converge only for $|z|>\left|z_{0}\right|$. On the other hand, we have seen that

$$
\lim _{z \rightarrow \infty} U_{1}^{\infty}(z) \sim e^{-i \omega z} z^{i \eta-\frac{B_{2}}{2}}, \quad \lim _{z \rightarrow \infty} \bar{U}_{1}^{\infty}(z) \sim e^{i \omega z} z^{-i \eta-\nu-\frac{B_{2}}{2}},
$$

where $\nu$ is the characteristic parameter of the two-sided series. The behaviour of $U_{1}$ when $z \rightarrow \infty$ is given by a combination of these. This explains why $U_{1}$ is sometimes inappropriate when the variable $z$ tends to infinity. In this event we need to use only one of the expansions in series of irregular functions.

All the solutions for the double-confluent Heun equation (DCHE), obtained from solutions of the CHE through the Leaver limit $\left(z_{0} \rightarrow 0\right)$, are also given by expansions in series of confluent hypergeometric functions. Then, the analysis used for the solutions of the CHE has been promptly adapted to the solutions of the DCHE, including the conditions which allow to write one solution of a fixed set as a linear combination of the others.

We have also found one-sided infinite-series solutions for the CHE and DCHE, by truncating the two-side infinite series on the left. The one-sided solutions for the CHE can afford two different types of finite-series solutions. One of these may be ignored because they are also suplied by the Barber-Hassé expansions in power series. The other type of finite series is important because it is the only one preserved by the Whittaker-Ince limit of the CHE.

The Whittakker-Ince limit (5) of the CHE and DCHE have generated expansions in series of Bessel functions of the first kind and in series of the first and second Hankel functions. In this case, each solution belonging to fixed set can be written as a linear combination of the others without the need of restrictions on the parameters of the differential equations. The Whittakker-Ince limit of the DCHE does not admit finite-series solutions.

In order to have a full account of the solutions, we have considered the transformations rules which result from substitutions of variables which transform a Heun equation into another form of itself (up to a change of parameters). For instance, only if we apply the transformation rules to solutions of the CHE, can we derive solutions for the Mathieu and Whittaker-Hill equations having all the desired properties of parity and periodicity. Similarly, if an expansion is not valid for certain values of the parameters, the transformation rules allow to find another solution valid for that case.

Solutions in series of Coulomb wave functions [25] and in series of Gauss hypergeometric functions [14, 28, 29] for the confluent Heun equation also admit both the Leaver and the Whittaker-Ince limits [14, 15]. However, there are several unexplored aspects concerning such expansions. Thus, it is necessary to consider other solutions of this type in order to retrieve the usual solutions of the ordinary spheroidal equation [1, 2, 32, which is a particular case of Eq. (11) with $\eta=0$ [25]. This would amount new solutions for the equations of the diagram written in the first section. On the other side, by means of the Whittaker-Ince limit these solutions conduct to known solutions for the Mathieu equations [15. Nevertheless, the possibility of finding solutions for the CHE which reproduce all the known solutions of the Mathieu 
equations is an open issue.

Now we refer to some problems which obey the CHE and its limiting cases. In the first place, the Schrödinger equation with inverse fourth and sixth-power potentials leads, respectively, to the DCHE (3) and its Whittaker-Ince limit (7). These potentials have appeared, for example, in the description of intermolecular forces [17] and in the scattering of ions by polarisable atoms [24].

In fact, the radial equation for the scattering of ions by neutral targets with induced dipole and quadrupole moments can be put in the form of the WhittakerInce limit of the DCHE [15]; for neutral or charged targets presenting only induced dipole moment, the equation may be reduced to the DCHE [7, 15]. In these problems, there is no arbitrary constant in the differential equations and so, in order to assure the series convergence, it is necessary to use two-sided infinite series solutions with a characteristic parameter $\nu$.

In the second place, as already mentioned, the equations which rule the timedependence of Klein-Gordon and Dirac test-fields in some nonflat Friedmannian spacetimes can be written as CHEs and DCHEs [6, 14. These equations also require two-sided infinite series solutions. In addition, there are the CHEs for angular and radial Teukolsky equations in Kerr spacetimes [4, 25, 28, 29].

Finally, in Appendix A we have found that normal forms of the Heun equations are helpful to know if a given one-dimensional Schrödinger equation reduces to Heun equations. In this connection, we have seen that there are quasi-exactly solvable (QES) potentials representative of each of the five Heun equations, a fact which may become important if we have sufficient knowledge about the solutions of the equation.

As noticed at the beginning, QES problems demand finite-series solutions which give only a part of the energy spectrum, but in the case of Heun equations there are also infinite-series solutions. Whether these afford the remaining part of the spectrum, this is a theme to be analysed. At last, we observe that the inverted QES potential (A.7) leads to the Whittaker-Ince limit of the confluent Heun equation. As far as we are aware, the only other example known for this equation (excepting Mathieu equations) comes from the separation of variables of the Laplace-Beltrami operator for scalar fields in an Eguchi-Hanson space [5, 27, 31].

\section{Appendix A. Heun Equations and Quasiexact Solvability}

We consider the Heun equations in their normal or Schrödinger form,

$$
\left[\frac{d^{2}}{d z^{2}}+Q(z)\right] y(z)=0
$$

that is, in the form where there is no first-order derivative term [10]. The function $Q(z)$ for the general Heun equation and its confluent cases is given in the following [34], where it is understood that, in general, there exists some constraint among the parametres $A, B$, and so on. In each case we indicate the singular points of the equation.

General Heun equation. Four regular singular points at $z=0,1, a, \infty$ :

$$
Q(z)=\frac{A}{z}+\frac{B}{z-1}+\frac{C}{z-a}+\frac{D}{z^{2}}+\frac{E}{(z-1)^{2}}+\frac{F}{(z-a)^{2}}, \quad(a \neq 0 \text { or } 1) .
$$

Confluent Heun equation (or generalised spheroidal wave equation). Two regular points at $z=0,1$ and one irregular at $z=\infty$ :

$$
Q(z)=A+\frac{B}{z}+\frac{C}{z-1}+\frac{D}{z^{2}}+\frac{E}{(z-1)^{2}}
$$


Double-confluent Heun equation. Two irregular points at $z=0, \infty$ :

$$
Q(z)=A+\frac{B}{z}+\frac{C}{z^{2}}+\frac{D}{z^{3}}+\frac{E}{z^{4}} .
$$

Biconfluent Heun equation. One regular point at $z=0$ and one irregular point at $z=\infty$ :

$$
Q(z)=A z^{2}+B z+C+\frac{D}{z}+\frac{E}{z^{2}} .
$$

Triconfluent Heun equation. One irregular point at $z=\infty$ :

$$
Q(z)=A z^{4}+B z^{3}+C z^{2}+D z+E .
$$

Other normal forms are given in the tables constructed by Lemieux and Bose [26] who, however, have not considered the triconfluent equation. These tables are helpful to recognise whether a given equation is of the Heun type. In particular, by using the Lemieux-Bose tables in addition to the normal forms written above, it is straightforward to establish relations among the Heun and the Schrödinger equations for some quasi-exactly solvable potentials.

We find: (i) a triconfluent Heun equation for the quartic potential given in Eq. (5.34) of González-López, Kamran and Olver [18; (ii) biconfluent Heun equations for the sextic potential $V_{1} z^{6}+V_{2} z^{4}+V_{3} z^{2}+V_{4}+V_{5} / z^{2}$ given by Turbiner 38] and Ushveridze [40], and for the potentials II, III and VIII given in Turbiner's list [38]; (iii) double-confluent Heun equations for the inverse fourth-power potential $V(r)=V_{1} r^{-4}+V_{2} r^{-3}+V_{3} r^{-2}+V_{4} r^{-1}$ [37, 38], and for the asymmetric doubleMorse potential given by Zaslavskii and Ulyanov [44; (iv) confluent Heun equations for the trigonometric and hyperbolic potentials given by Ushveridze [39]; (v) general Heun equations in the Darboux elliptic form [9] for the first and second Ushveridze's elliptic potentials [39].

Finally we write a potential given by Cho and Ho [8], namely,

$$
V(u)=-\frac{\mathrm{b}^{2}}{4} \sinh ^{2} u-\left(\ell^{2}-\frac{1}{4}\right) \frac{1}{\cosh ^{2} u}, \quad u \in(-\infty, \infty), \quad(\ell=1,2,3, \cdots)
$$

where $\mathrm{b}$ is a positive real constant. This is a bottomless potential in the sense that $V(u) \rightarrow-\infty$ when $u \rightarrow \pm \infty$. If $\mathrm{b}^{2}<4 \ell^{2}-1$, it is an inverted double-well potential; if $\mathrm{b}^{2} \geq 4 \ell^{2}-1$, the potential is similar to the one of an inverted oscillator. For this potential the Schrödinger reduces to the Whittaker-Ince limit (6) of the confluent Heun equation.

\section{Appendix B. Confluent Hypergeometric Functions}

The regular and irregular confluent hypergeometric functions, denoted respectively by $\Phi(a, c ; y)$ and $\Psi(a, c ; y)$, satisfy the Kummer transformations

$$
\Phi(\mathrm{a}, \mathrm{c} ; y)=e^{y} \Phi(c-a, c ;-y), \quad \Psi(a, c ; y)=y^{1-c} \Psi(1+a-c, 2-c ; y) .
$$

The behaviour of of $\Psi(a, c ; y)$ and $\Phi(a, c ; y)$ when $y \rightarrow \infty$ is given by

$$
\begin{aligned}
& \lim _{y \rightarrow \infty} \Psi(a, c ; y) \sim y^{-a}\left[1+O\left(|y|^{-1}\right)\right], \quad\left(-\frac{3 \pi}{2}<\arg y<\frac{3 \pi}{2}\right) \\
& \lim _{y \rightarrow \infty} \Phi(a, c ; y)=\left\{\begin{array}{lr}
\frac{\Gamma(c)}{\Gamma(a)} e^{y} y^{a-c}\left[1+O\left(|y|^{-1}\right)\right], & (\Re y>0) \\
\frac{\Gamma(c)}{\Gamma(c-a)}(-y)^{-a}\left[1+O\left(|y|^{-1}\right)\right], & (\Re y<0) .
\end{array}\right.
\end{aligned}
$$


These can be used to get the limits of the series expansions as $y \rightarrow \infty$. For $\Re y=0$, the limit of $\Phi(a, c ; y)$ is a combination of the limits on the right-hand side of the previous expression [12].

To find the expansions in series of irregular confluent hypergeometric functions for the CHE, in addition to Eq. (23), we need the relations

$$
\begin{aligned}
& y \frac{d \Psi(a, c ; y)}{d y}=(1-c) \Psi(a, c ; y)+(c-a-1) \Psi(a, c-1 ; y), \\
& \frac{d \Psi(a, c ; y)}{d y}=\Psi(a, c ; y)-\Psi(a, c+1 ; y), \\
& (c-a-1) \Psi(a, c-1 ; y)+(1-c-y) \Psi(a, c ; y)+y \Psi(a, c+1 ; y)=0,
\end{aligned}
$$

where the last relation results from the first and second ones. These relations also hold for the functions $\tilde{\Phi}(a, b ; y)$ defined in Eq. (32). On the other hand, if $c \rightarrow \infty$, while $a$ and $y$ remain bounded, we have 12

$$
\begin{aligned}
& \Psi(a, c ; y)=(c)^{-a}\left[(-1)^{-a}+(\sqrt{2 \pi} / \Gamma(a)) c^{c+a-(3 / 2)} y^{1-c} e^{y-c}\right]\left[1+O\left(|c|^{-1}\right)\right] \\
& =(c)^{-a}\left[(-1)^{-a}+\frac{\sqrt{2 \pi}}{\Gamma(a)}\left(\frac{c}{e y}\right)^{c+a-(3 / 2)} y^{a-(1 / 2)} e^{y+a-(3 / 2)}\right]\left[1+O\left(\frac{1}{|c|}\right)\right]
\end{aligned}
$$

which, in conjunction with the last realion of Eq. (B.4), is useful in the study of the convergence of the series solutions for the CHE. Notice that this equation implies

$$
\begin{aligned}
& \lim _{c \rightarrow-\infty} \Psi(a, c ; y) \sim(-c)^{-a}\left[1+O\left(|c|^{-1}\right)\right], \\
& \lim _{c \rightarrow+\infty} \Psi(a, c ; y) \sim \frac{\sqrt{2 \pi}}{\Gamma(a)}\left(\frac{c}{e y}\right)^{c+a-\frac{3}{2}} c^{-a} y^{a-\frac{1}{2}} e^{y+a-(3 / 2)}\left[1+O\left(\frac{1}{|c|}\right)\right] .
\end{aligned}
$$

The Whittaker-Ince limit of the expansions in series of confluent hypergeometric functions for the CHE and DCHE yields expansions in series of Bessel functions for Eqs. (6) and (7) by means of the limits [12]

$$
\begin{aligned}
& \lim _{a \rightarrow \infty} \Phi\left(a, c ;-\frac{y}{a}\right)=\Gamma(c) y^{(1-c) / 2} J_{c-1}(2 \sqrt{y}), \\
& \lim _{a \rightarrow \infty}\left[\Gamma(a+1-c) \Psi\left(a, c ; \frac{y}{a}\right)\right]=2 y^{(1-c) / 2} K_{c-1}(2 \sqrt{y}), \\
& \lim _{a \rightarrow \infty}\left[\Gamma(a+1-c) \Psi\left(a, c ;-\frac{y}{a}\right)\right]=\left\{\begin{array}{l}
-i \pi e^{i \pi c} y^{(1-c) / 2} H_{c-1}^{(1)}(2 \sqrt{y}), \operatorname{Im} y>0, \\
i \pi e^{-i \pi c} y^{(1-c) / 2} H_{c-1}^{(2)}(2 \sqrt{y}), \operatorname{Im} y<0,
\end{array}\right.
\end{aligned}
$$

where $J_{\lambda}$ denotes the Bessel functions of the first kind of order $\lambda, K_{\lambda}$ are modified Bessel functions of the third kind, whereas $H_{\lambda}^{(1)}$ and $H_{\lambda}^{(2)}$ denote the first and second Hankel functions [13], respectively. Some relations useful for Sec. 3.2 are

$$
\begin{array}{ll}
H_{-\lambda}^{(1)}(x)=e^{i \pi \lambda} H_{\lambda}^{(1)}(x), & H_{-\lambda}^{(2)}(x)=e^{-i \pi \lambda} H_{\lambda}^{(2)}(x), \\
K_{\lambda}(-i x)=\frac{1}{2} \pi i e^{\frac{1}{2} \pi i \lambda} H_{\lambda}^{(1)}(x), & K_{\lambda}(i x)=-\frac{1}{2} \pi i e^{-\frac{1}{2} \pi i \lambda} H_{\lambda}^{(2)}(x) .
\end{array}
$$

\section{References}

[1] M. Abramowitz and I. A. Stegun (eds.), Handbook of Mathematical Functions (Dover, New York, 1965). 
[2] F. M. Arscott, Periodic Differential Equations (Oxford: Pergamon Press, 1964).

[3] W. G. Barber and H. R. Hassé, The two centre problem in wave mechanics, Proceedings of Cambridge Philosophical Society 25 (1935) 564-581.

[4] D. Bini, C. Cherubini, R. T. Jantzen and B. Mashhoon, Massless field perturbations and gravitomagnetism in the Kerr-Taub-NUT spacetime, Phys. Rev. D67 (2003) 084013.

[5] T. Birkandan and M. Hortaçsu, Comment on 'Dirac equation in the background of the Nutku helicoid metric', J. Math. Phys. 49 (2008) 054101.

[6] N. D. Birrel and P. C. W. Davies, Quantum Fields in Curved Spaces (Cambridge University Press, 1982).

[7] W. Bühring, Schrödinger equation with inverse fourth-power potential, a differential equation with two irregular singular points, J. Math. Phys. 15 (1974) 1451-1459.

[8] H. T. Cho and C. L. Ho, A novel quasi-exactly solvable model with total transmission modes, J. Phys. A: Math. Theor. 41 (2008) 172002; e-print arXiv:quant-ph/0606144v3.

[9] G. Darboux, Sur une équation linéaire, Comp. Rendu XCIV 25 (1882) 1645-1650.

[10] A. Decarreau, P. Maroni and A. Robert, Sur les équations confluentes de l'équation de Heun, Ann. Soc. Sci. Bruxelles T92(III) (1978) 151-189.

[11] A. Decarreau, M. C. Dumont-Lepage, P. Maroni, A. Robert and A. Ronveaux, Formes canoniques des équations confluentes de l'équation de Heun, Ann. Soc. Sci. Bruxelles T92(I-II) (1978) 53-78.

[12] A. Erdélyi, W. Magnus, F. Oberhettinger and F. G. Tricomi, Higher Transcendental Functions, Vol. 1 (McGraw-Hill, New York, 1953).

[13] A. Erdélyi, W. Magnus, F. Oberhettinger and F. G. Tricomi, Higher Transcendental Functions, Vol. 2 (McGraw-Hill, New York, 1953).

[14] B. D. B. Figueiredo, On some solutions to generalized spheroidal wave equations and applications, J. Phys. A: Math. Gen. 35 (2002) 2877-2906.

[15] B. D. B. Figueiredo, Ince's limits for confluent and double-confluent Heun equations, $J$. Math. Phys. 46 (2005) 113503.

[16] B. D. B. Figueiredo, Generalized spheroidal wave equation and limiting cases, J. Math. Phys. 48 (2007) 013503.

[17] W. M. Frank, D. J. Land and R. M. Spector, Singular potentials, Rev. Mod. Phys. 43 (1971) 36-98.

[18] A. González-López, N. Kamran and P. J. Olver, Normalizability of one-dimensional quasi-exactly solvable Schrödinger operators, Commun. Math. Phys. 153 (1993) 117146.

[19] P. Humbert, Fonctions de Lamé et fonctions de Mathieu, Mémorial des Sciences Mathématiques Vol. X (Gauthier-Villards, Paris, 1926).

[20] E. A. Hylleraas, Über die Elektronenterme des Wasserstoffmoleküls, Z. Phys. 71 (1931) 739-763.

[21] E. L. Ince, A linear differential equation with periodic coefficients, Proceedings of London Mathematical Society 23 (1923) 56-74.

[22] G. Jaffé, Zur Theorie des Wasserstoffmolekülions, Z. Phys. 87 (1934) 535-544.

[23] E. G. Kalnins, W. Miller and G. S. Pogosyan, Exact and quasiexact solvability of secondorder superintegrable quantum systems: I. Euclidian space preliminaries, J. Math. Phys. 47 (2006) 033502. 
[24] C. J. Kleinman, Y. Hahn and L. Spruch, Dominant nonadiabatic contribution to the long-range electron-atom interaction, Phys. Rev. 165 (1968) 53-62.

[25] E. W. Leaver, Solutions to a generalized spheroidal wave equation: Teukolsky equations in general relativity, and the two-center problem in molecular quantum mechanics, $J$. Math. Phys. 27 (1986) 1238-1265.

[26] A. Lemieux and A. K. Bose, Construction de potentiels pour lesquels l'équation de Schrödinger est soluble, Ann. Inst. Henri Poincaré Sect. A 10 (1969) 259-270.

[27] A. Malmendier, The eigenvalue equation on the Eguchi-Hanson space, J. Math. Phys. 44 (2003) 4308-4343.

[28] S. Mano, H. Suzuki and E. Takasugi, Analytic solutions of the Teukolsky equation and their low frequency expansions, Prog. Theor. Phys. 95(6) (1996) 1079- 1096.

[29] S. Mano, H. Suzuki and E. Takasugi, Analytic solutions of the Regge-Wheeler equation and the post-Minkowskian expansion, Prog. Theor. Phys. 96(3) (1996) 549-565.

[30] E. W. McLachlan, Theory and Application of Mathieu Functions (Dover, New York, 1964).

[31] S. Mignemi, Classical and quantum motion in an Eguchi-Hanson space, J. Math. Phys., 32 (1991) 3047-3054.

[32] J. Meixner and F. W. Schäfke, Mathieusche Funktionen und Sphäroidfunctionen (SpringVerlag, Berlin, 1954).

[33] F. M. J. Olver, Asymptotics and Special Functions (Academic Press, New York, 1974).

[34] A. Ronveaux (ed.), Heun's Differential Equations (Oxford University Press, New York, 1995).

[35] E. Schrödinger, Eigenschwingungen des sphärischen Raumes, Commentationes Pontificiae Academiae Scientiarum 2 (1938) 321-364.

[36] E. Schrödinger, Maxwell's and Dirac's equations in the expanding universe, Proceedings of Royal Irish Academy 2 (1940) 25-47.

[37] A. V. Turbiner, Quantum mechanics: problems intermediate between exactly solvable and completely unsolvable, Sov. Phys. JETP 67 (1988) 230-236.

[38] A. V. Turbiner, Quasi-exactly-solvable problems and sl(2) algebra, Commun. Math. Phys. 118 (1988) 467-474.

[39] A. G. Ushveridze, Quasi-exactly solvable models in quantum mechanics, Sov. J. Part. Nucl. 20 (1989) 504-528.

[40] A. G. Ushveridze, Quasi-Exactly Solvable Models in Quantum Mechanics (IOP Publishing, Bristol, 1994).

[41] E. T. Whittaker and G. N. Watson, A Course of Modern Analysis (Cambridge University Press, 1945).

[42] A. H. Wilson, A generalised spheroidal wave equation, Proc. Roy. Soc. of London A118 (1928) 617-635.

[43] A. H. Wilson, The ionised hydrogen molecule, Proc. Roy. Soc. of London A118 (1928) 635-647.

[44] O. B. Zaslavskii and V. V. Ulyanov, New classes of exact solutions of the Schrödinger equation and the potential-field description of spin systems, Sov. Phys. JETP 60 (1984) 991-996. 